\title{
« Mon père pensait autrement »: des garçons nigérians réfléchissent à l'égalité entre les sexes
}

Françoise Girard

Follow this and additional works at: https://knowledgecommons.popcouncil.org/departments_sbsr-pgy

Part of the Family, Life Course, and Society Commons, Gender and Sexuality Commons, International Public Health Commons, and the Public Health Education and Promotion Commons How does access to this work benefit you? Let us know!

\section{Recommended Citation}

Girard, Françoise. 2003. "« Mon père pensait autrement » : des garçons nigérians réfléchissent à l'égalité entre les sexes," Quality/Calidad/Qualité no. 14. New York: Population Council. 
«Mon père pensait autrement » : des garçons nigérians réfléchissent à l'égalité entre les sexes
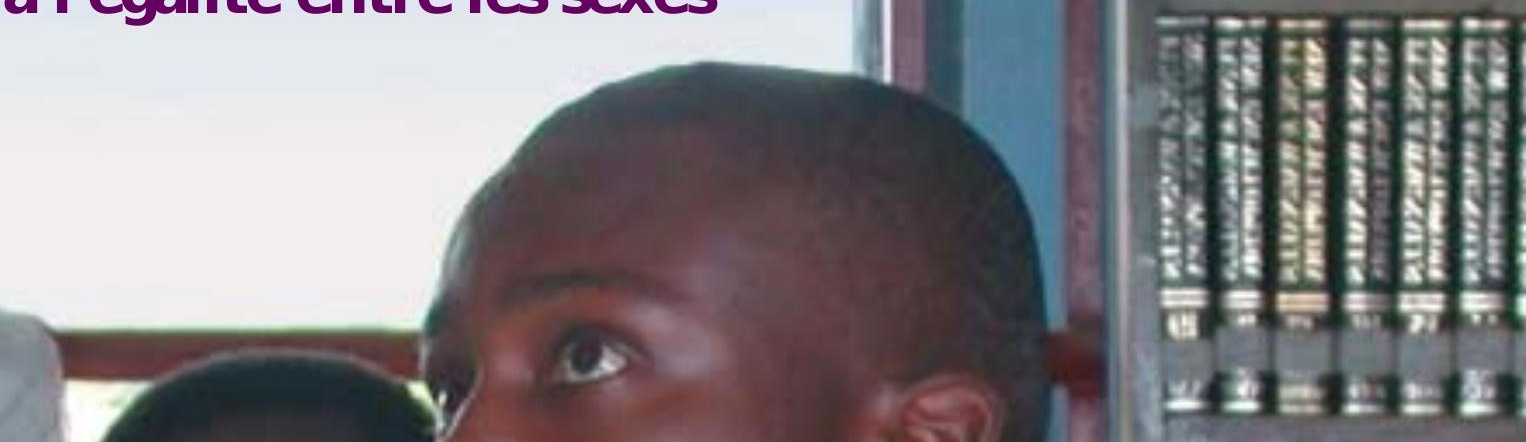


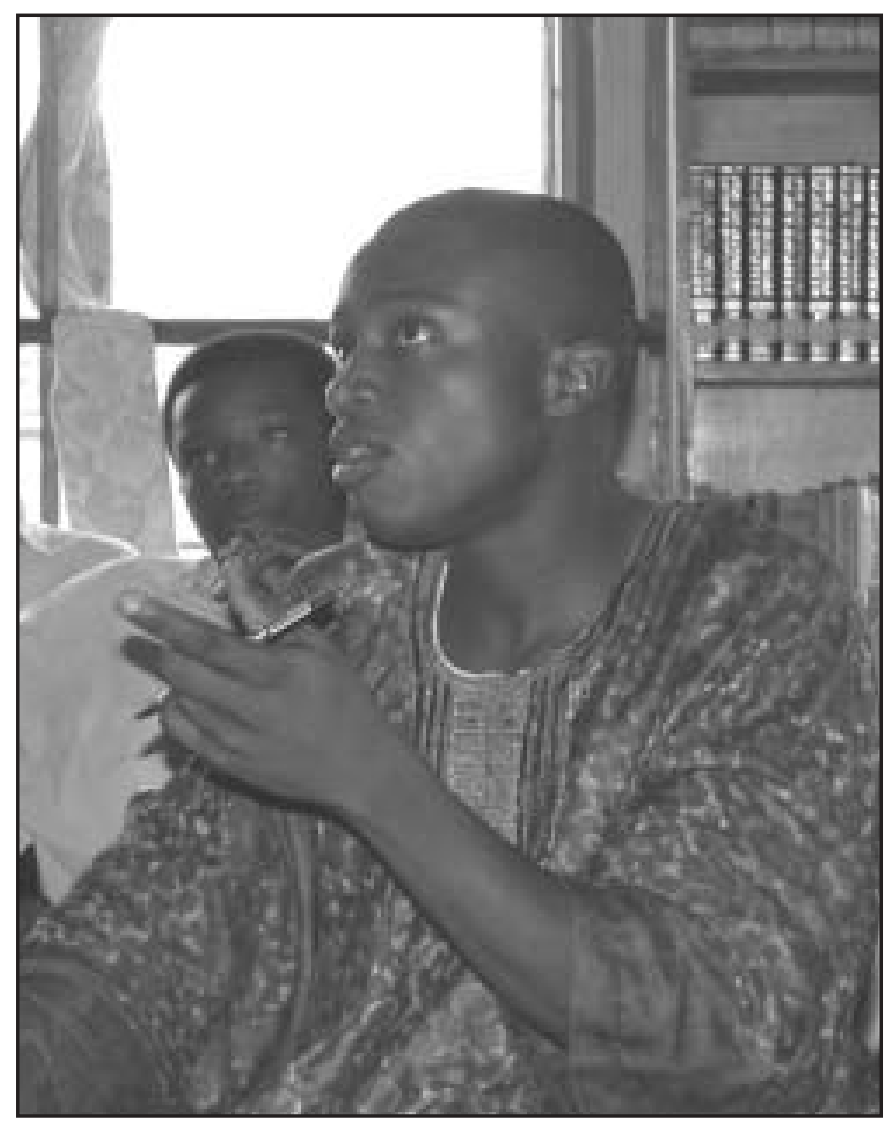

«Mon père pensait autrement 》: des garçons nigérians réfléchissent à l'égalité entre les sexes 
Quality/Calidad/Q ualité, une publication du Population Council, met en lumière des exemples de programmes cliniques et éducatifs qui témoignent de leur engagement résolu à fournir des soins de haute qualité en santé sexuelle et reproductive et qui emploient des approches novatrices et réfléchies. La série est fondée sur la philosophie selon laquelle toute personne a fondamentalement droit à un traitement respectueux de la part des prestataires de soins de santé reproductive ainsi qu'à l'information, au choix et au suivi des soins.

$\mathrm{Q} / \mathrm{C} / \mathrm{Q}$ documente des projets qui font d'importants progrès d'une ou de plusieurs des manières suivantes : en élargissant le choix de méthodes et de technologies disponibles; en fournissant l'information dont les clients ont besoin pour opérer des choix éclairés; en permettant aux clients de devenir des gardiens plus efficaces de leur santé sexuelle et reproductive; en faisant des efforts novateurs pour accroître la capacité de gestion et développer les compétences des prestataires de services à tous les niveaux; en combinant les soins de santé, la planification familiale et les services connexes de manières novatrices et en s'efforçant d'atteindre des groupes mal desservis et défavorisés.

Les projets documentés sont choisis par un groupe consultatif composé d'individus possédant une vaste expérience en matière de promotion de la qualité des soins de santé sexuelle et reproductive. Aucun de ces projets n'est offert comme un modèle à reproduire. Chacun d'entre eux est plutôt présenté comme un exemple de valeurs, d'objectifs et d'exécution révélant une créativité exceptionnelle. Ils représentent des expériences d'apprentissage qui illustrent l'attitude autocritique requise pour anticiper les besoins des clients et trouver des moyens abordables de les satisfaire. Cette attitude réfléchie se manifeste par la volonté des projets de répondre aux changements qui surviennent dans les besoins des clients ainsi qu'aux plus grandes transformations économiques et sociales qui affectent les sociétés. La documentation des choix critiques faits par ces programmes devrait aider à renforcer, en pratique, la conviction que la satisfaction de l'individu à l'égard des services de santé sexuelle et reproductive est étroitement liée à la réalisation de buts sanitaires et démographiques plus larges.

Cette édition française de Q uality/Calidad/Q ualité a été publiée grâce au soutien de I'International Women's Health Coalition, de la Fondation Ford, du programme régional pour l'Afrique du Population Council, ainsi que du programme « Genre, famille et développement » du Population Council. Les déclarations faites dans cette publication ainsi que les opinions exprimées sont I'entière responsabilité des auteurs et non d'une quelconque organisation soutenant $Q / C / Q$. Toute partie de ce document peut être reproduite sans la permission des auteurs, pourvu que ce ne soit pas à des fins lucratives.

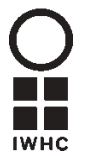

L'International Women's Health Coalition développe et finance des politiques et programmes sanitaires et démographiques qui visent la promotion et la protection des droits ainsi que de la santé des filles et femmes du monde entier, particulièrement en Afrique, en Asie, en Amérique latine et dans les pays de la transition post-socialiste.

\section{$(1$ Population Council}

Le Population Council est une organisation non gouvernementale internationale, à but non lucratif, qui cherche à améliorer le bien-être et la santé reproductive des générations actuelles et futures dans le monde entier, et à favoriser un équilibre humain, équitable et durable entre personnes et ressources. L'organisme mène des recherches dans le domaine biomédical, des sciences sociales et de la santé publique, et il aide à développer les capacités de recherche dans les pays en développement. Fondé en 1952, le Population Council est dirigé par un conseil d'administration international. Son siège, qui se trouve à $\mathrm{N}$ ew York, soutient un réseau mondial de bureaux régionaux et nationaux.

Population Council, O ne Dag Hammarskjold Plaza, New York, N ew York 10017 USA

téléphone: 212-339-0500 fax: 212-755-6052 courriel:qcq@popcouncil.org http://www.popcouncil.org

Photographie de couverture : Corinne W hitaker. La photo de la page 2 provient de M /M C Photoshare à l'adresse suivante : www.jhuccp.org/mmc.

Publication N 14 ISSN : 1097-8194

Copyright $\odot 2003$ The Population Council, Inc. 
de G ary Barker

La « participation des hommes » a commencé à susciter l'attention dans le domaine de la population et de la planification familiale dans les années 1980, essentiellement parce que ce sont les hommes qui décidaient souvent de la taille de la famille et de l'emploi des contraceptifs. Deux décennies de lutte contre la violence sexuelle et le VIH ont cependant permis de tirer une leçon indéniable, à savoir que fréquemment, ce sont aussi les hommes qui décident si les femmes et les filles doivent avoir des rapports sexuels, et sous quelles conditions. Progressivement, les planificateurs de programme, les organisations de développement international et les chercheurs ont fini par comprendre que les inégalités entre les sexes-dans les relations sexuelles, la vie conjugale et la société en général- sont la cause première des mauvais résultats enregistrés dans le domaine de la santé sexuelle et reproductive des hommes et des femmes. En 1994, les délégués de 180 pays, réunis au Caire dans le cadre de la Conférence internationale sur la population et le développement, ont affirmé que les hommes doivent être inclus dans les efforts visant à améliorer non seulement la santé reproductive des femmes, mais aussi le statut social et juridique des femmes et filles du monde entier (Organisation des Nations Unies 1995).

Certains programmes visant les hommes se focalisent principalement sur les adolescents plutôt que sur les hommes adultes. Une des raisons invoquées est que les adolescents ont des besoins particuliers. Beaucoup d'entre eux ont du mal à continuer leurs études ou à réussir à l'école, à trouver du travail ou à subvenir à leurs propres besoins ainsi qu'à ceux de leur famille. Les adolescents plus âgés traversent la phase de leur vie pendant laquelle ils courent le risque de contracter des maladies sexuellement transmissibles (y compris le VIH), et sont exposés aux risques liés à la violence. Par exemple, les

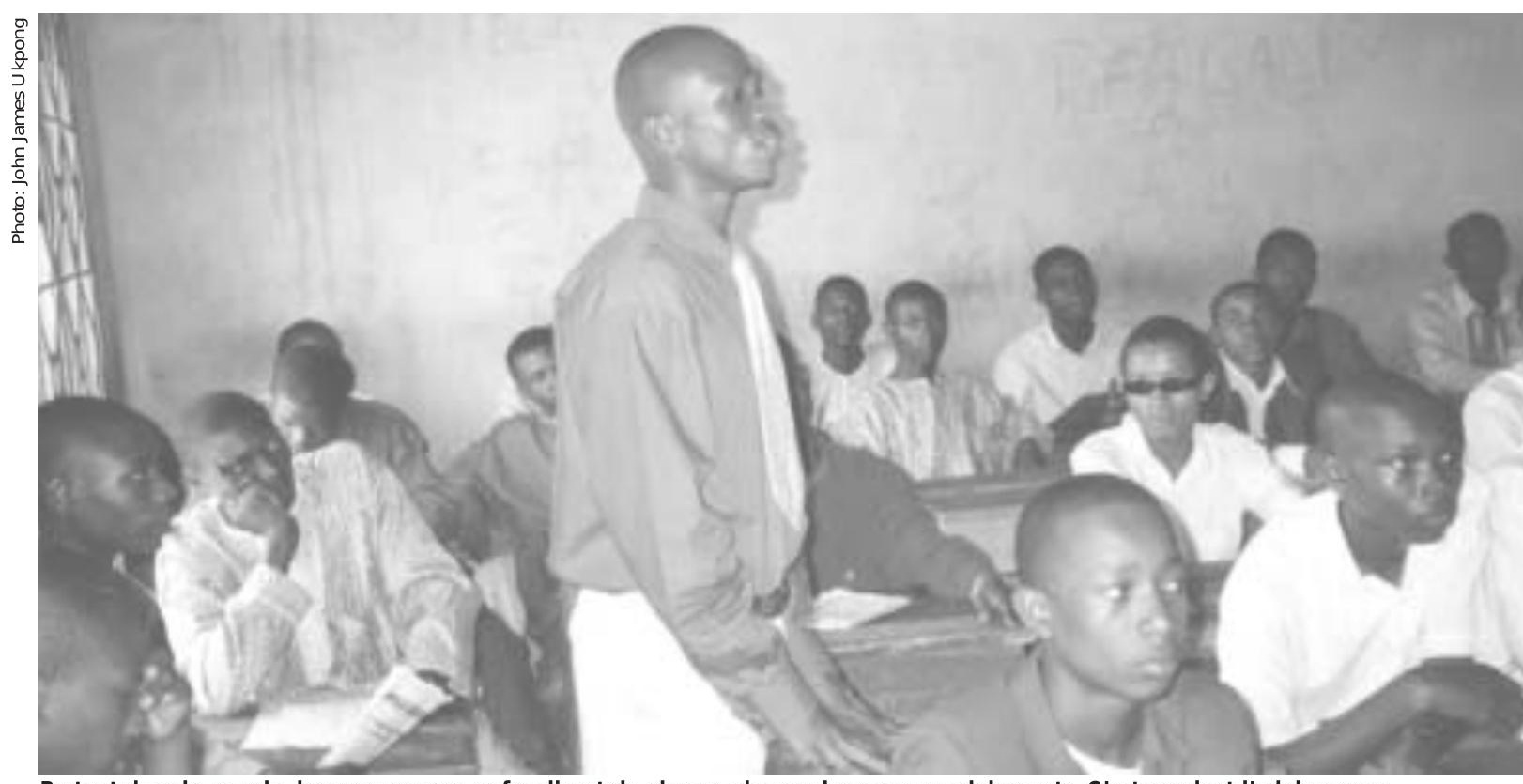

Partout dans le monde, les programmes se focalisent de plus en plus sur les garçons adolescents. C'est pendant l'adolescence que les garçons « apprennent à être des hommes », à absorber et intérioriser les rôles et style d'interaction dans les relations intimes qu'ils joueront et auxquels ils se conformeront probablement au cours de toute leur vie d'adulte. 
taux de mortalité chez les jeunes hommes âgés de 15 à 24 ans sont de deux à cinq fois supérieurs à ceux des jeunes femmes de la même tranche d'âge, et considérablement plus élevés que ceux des hommes adultes. La majorité de ces décès prématurés sont dus aux homicides et aux accidents de la circulation (Barker 2000). Une autre raison justifiant la priorité accordée aux programmes pour adolescents est que c'est pendant l'adolescence que les garçons apprennent à être des

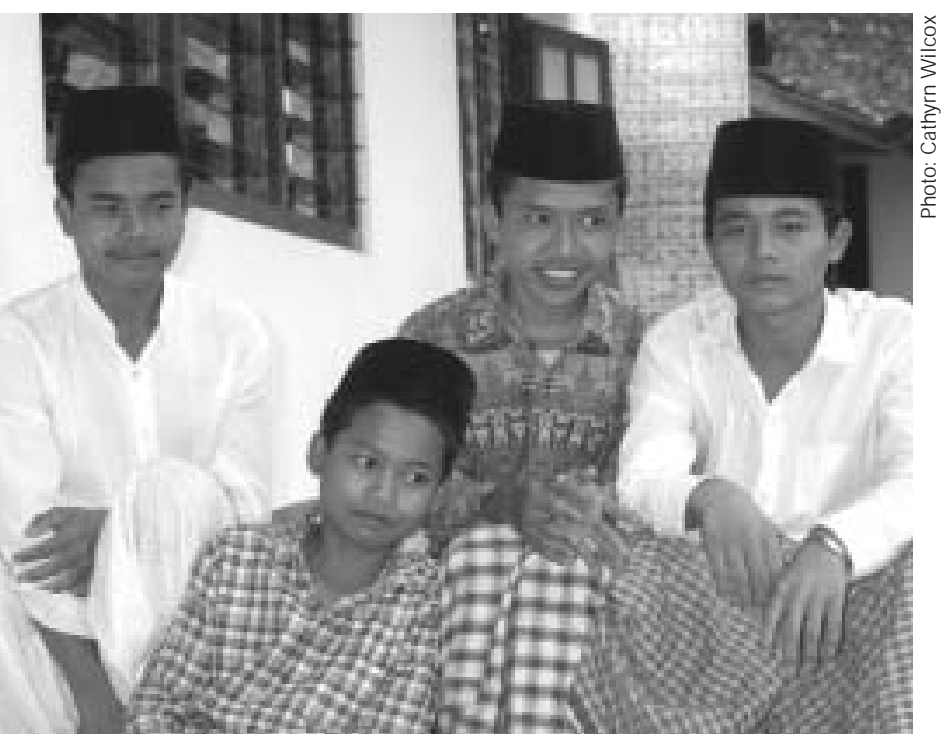

L'une des leçons émergeant des programmes pour adolescents dans le monde entier concerne la nécessité de créer des cadres où les jeunes hommes peuvent remettre en question ce qui est souvent considéré comme incontestable, par exemple ce que signifie «être un homme».

hommes, à absorber et intérioriser les rôles et style d'interaction dans les relations intimes qu'ils joueront et auxquels ils se conformeront probablement au cours de toute leur vie d'adulte.

Presque toutes les cultures et familles encouragent un genre de masculinité extravertie, orientée vers la réussite, qui a pour but de faire des garçons des pourvoyeurs et des protecteurs (Gilmore 1990). $D$ ans de nombreux pays, les garçons, dès leur jeune âge, passent généralement plus de temps dans la rue ou hors de la maison sans surveillance que les filles qui, elles, sont souvent contraintes à l'isolement social et l'exclusion pendant l'adolescence (Barker 2000). L'entourage familial et le groupe de pairs masculins sont les lieux où les garçons et les jeunes hommes « s'essayent aux rôles macho et répètent ces rôles » (Mosher et Tomkins 1988). La socialisation de nombreux jeunes garçons leur impose d'être agressifs et violents et d'avoir l'esprit de compétition, caractéristiques considérées comme utiles pour des pourvoyeurs et des protecteurs, tandis que les filles apprennent à ne pas être violentes et à parfois accepter passivement la violence et l'initiative sexuelle des hommes (Archer 1984). Dans certains contextes, les garçons apprennent à adhérer à des codes rigides d'honneur et de courage les obligeant à rivaliser, se battre et recourir à la violence, même lors d'altercations mineures (Archer 1994). Beaucoup de garçons en viennent à concevoir les rapports sexuels en ces mêmes termes, ils apprennent à considérer les filles et les femmes comme des objets de leur plaisir sexuel, à user de la force pour avoir des rapports sexuels et à envisager les rapports sexuels comme une simple performance. Pour établir leur masculinité, les jeunes hommes dans la plupart des pays du monde doivent aussi indiquer qu'ils sont hétérosexuels afin d'éviter la stigmatisation sociale et l'isolement. L'homophobie est utilisée pour forcer les jeunes hommes (à la fois les hétérosexuels et ceux qui sont attirés par d'autres hommes) à se conformer à la norme. Pour ces raisons, les garçons prouvent leur masculinité en partie en racontant à leurs pairs qu'ils ont « réussi à coucher » avec des filles.

Si les groupes de pairs masculins contribuent à construire des versions traditionnelles négatives de la masculinité, ils peuvent aussi jouer un rôle dans la démolition de ces aspects néfastes des spécificités sexuelles. En effet, lorsque l'occasion leur en est donnée, les garçons sont capables de préciser ce qu'il en coûte pour réussir dans les rôles traditionnels masculins ou être fidèle à ces rôles. De plus, plusieurs études ont montré que chez certains jeunes hommes, le fait de réfléchir à leur exposition à des formes négatives de comportement masculin, telles que l'utilisation de la violence par leur père, est un puissant moyen d'engen- 
drer un changement d'attitude (Barker 2001). Q uand les garçons apprennent à respecter leurs partenaires et à pratiquer une activité sexuelle prudente, ils gardent souvent ce comportement jusqu'à l'âge adulte. Par exemple, une étude aux ÉtatsUnis a révélé que les adolescents qui avaient utilisé des préservatifs lors de leurs premiers rapports sexuels étaient plus susceptibles de les utiliser régulièrement par la suite (Sonenstein et al. 1995).

Ces conclusions suggèrent la nécessité de commencer à travailler avec les jeunes hommes plus tôt. Ainsi, après plusieurs années d'exhortation à « commencer plus tôt », un nombre croissant d'organisations à travers le monde ont conçu des programmes qui font participer de plus jeunes garçons à des discussions sur les questions liées à la santé et aux spécificités sexuelles. La poussée de croissance et la maturation physiologique associées à la puberté s'accompagnent d'un épanouissement des capacités de pensée critique et d'abstraction, y compris l'analyse des questions liées à la justice et la réflexion sur ces questions. Lors d'une étude menée en 1999 pour le compte de l'O rganisation mondiale de la santé, 77 programmes gouvernementaux et non gouvernementaux engageant les garçons et les jeunes hommes dans les écoles, les communautés de base, les lieux de travail, les installations militaires et les tribunaux pour enfants ont été consultés (Barker 2000). Leurs activités comprennent, entre autres, I'offre dans les centres de santé d'heures de consultation destinées aux garçons, la mise en rapport des garçons avec des mentors qui sont des modèles de comportement masculin positifs, la formation professionnelle et l'organisation d'activités de vulgarisation en matière de santé sexuelle. Dans la majorité des programmes, certains thèmes sont abordés au sein de groupes constitués uniquement de garçons, tandis que garçons et filles sont regroupés pour discuter de l'inégalité sexiste. Certains programmes, comme la White Ribbon Campaign (campagne du ruban blanc), une campagne internationale menée par des hommes luttant pour mettre fin à la violence contre les femmes, cherchent à inclure à la fois les garçons et les hommes adultes.

Plusieurs leçons émergent de cette étude ainsi que des études de cas menées plus en profondeur. Dans l'ensemble, l'expérience révèle que les programmes doivent adopter les priorités suivantes: découvrir les raisons pour lesquelles les garçons veulent changer, impliquer relativement peu de jeunes hommes dans de petits groupes de discussion intensive pendant une période prolongée, exploiter

\section{À mesure que la conception des programmes devient plus sophis- tiquée, nous devons élaborer de nouveaux instruments de mesure capables de saisir des changements significatifs dans les attitudes et les comportements, même quand ces changements sont déterminés par de multiples facteurs.}

le pouvoir positif des groupes de pairs masculins pour encourager l'équité entre les sexes, aborder le thème de I'homophobie, planifier des activités qui réclament un effort énergique de la part des participants et qui concernent de multiples thèmes, travailler avec les garçons sur la question des soins personnels et de la prévention et créer des cadres dans lesquels les jeunes hommes peuvent formuler ouvertement leurs doutes et remettre en question les sujets souvent considérés comme incontestables (par exemple ce que signifie « être un homme »).

Un grand nombre de ces leçons sont confirmées par l'étude de cas suivante dans laquelle Françoise Girard décrit le programme nigérian « Conscientiser les adolescents de sexe masculin » (CMA, Conscientizing Male Adolescents). Contrairement à beaucoup de programmes visant les adolescents de sexe masculin, qui mettent l'accent sur des activités à court terme destinées à prévenir la maladie et les grossesses non désirées, le 
modèle proposé par CMA est un programme à long terme axé sur le sexisme et le développement de la pensée critique.

Les garçons se réunissent hebdomadairement pour discuter de sujets comme l'oppression sexiste, les droits sexuels, la violence, le pouvoir au sein de la famille, les relations intimes, la santé sexuelle, les droits de la personne et la démocratie. Pour aider les garçons à développer un mode de pensée critique, les enseignants ont beaucoup recours au dialogue socratique et enseignent la logique ainsi que l'expression orale. Plutôt que de viser les jeunes gens déscolarisés, à plus haut risque, CMA recrute des participants qui vont à l'école, sont particulièrement intelligents et font figure de leaders sociaux. L'engagement audacieux des enseignants en faveur d'un travail intense et de longue haleine et d'une approche fondée explicitement sur les droits de la personne témoigne d'une vision claire et courageuse. Leur succès à ce jour offre de précieuses leçons sur la façon d'aider les jeunes hommes à assurer la transition à un âge adulte sain.

CMA a pris des mesures initiales pour évaluer le programme. Tous les programmes cherchant à changer la dynamique entre genre et pouvoir qui sous-tend de nombreux comportements associés à la santé sont confrontés à des défis quant à l'évaluation de leur efficacité. Le changement des attitudes individuelles et des normes sociales relatives aux rôles sexuels est un projet à long terme dif- ficile à mesurer. Lors de tout travail axé sur les jeunes gens, le processus de maturation introduit un facteur déconcertant supplémentaire : les jeunes gens se développant et changeant constamment, il est difficile de distinguer les changements survenus à la suite de l'intervention de ceux qui résultent de leur maturation naturelle. A mesure que la conception des programmes devient plus sophistiquée, nous devons élaborer de nouveaux instruments de mesure capables de saisir des changements significatifs dans les attitudes et les comportements, même quand ces changements sont déterminés par de multiples facteurs. Q uelques programmes visant les garçons ont commencé ce processus. En Amérique latine, une coalition de quatre ONG (Instituto PROMUNDO, ECOS et Programa PAPAI au Brésil, et Salud y Género au Mexique) a mis en œuvre le Projet $\mathrm{H}$, un modèle basé à la fois sur des conceptions théoriques et sur les résultats de travaux de recherche, qui illustre comment impliquer les jeunes hommes dans la promotion de la santé et de l'équité entre les sexes, avec un modèle d'évaluation conçu pour mesurer les changements d'attitude et de comportement. ${ }^{1}$

Les exemples de CMA et du Projet $\mathrm{H}$, entre autres, confirment que nous savons comment interpeler les jeunes hommes et que les attitudes sexistes historiquement renforcées peuvent changer. L'étape suivante consiste à intégrer de telles approches à une programmation à grande échelle par l'intermédiaire des écoles, des

1 Le Projet $\mathrm{H}$ est né au Brésil à la suite de travaux de recherche conduits dans un contexte spécifique pour évaluer qualitativement un continuum de comportements et d'attitudes montrant chez les jeunes hommes un souci plus ou moins marqué d'équité entre les sexes (Barker 2001). En se fondant sur cette recherche et sur les expériences collectives des quatre ONG, les organisations partenaires ont élaboré une série d'activités éducatives de groupe (en même temps que des activités communautaires pour promouvoir le changement des normes sociales) afin d'engager la discussion avec les jeunes hommes sur l'équité entre les sexes. L'essai du matériel dans six pays de la région a confirmé un changement qualitatif des attitudes concernant les spécificités sexuelles. L'évaluation de l'impact, qui est en cours, se focalise sur une série de questions concernant les attitudes-certaines affirmations des partisans de la masculinité traditionnelle, certaines autres traduisant des vues plus soucieuses d'équité entre les sexesquestions élaborées et posées à un échantillon aléatoire de jeunes hommes et d'hommes adultes. L'essai a montré non seulement que l'échelle était cohérente et valable, mais aussi qu'elle était en étroite corrélation avec un nombre de variables comportementales clés (y compris l'aveu volontaire d'avoir utilisé un préservatif lors du dernier rapport sexuel et d'avoir fait preuve de violence envers sa partenaire). Le modèle d'évaluation, qui mesure chez les hommes les attitudes favorables à l'équité entre les sexes (appelé GEM Scale, Gender-Equitable Attitudes in Men), est actuellement utilisé dans une étude d'évaluation d'impact à grande échelle. 
communautés de base, des lieux de travail et des familles. Prendre au sérieux les rôles sexuels et les iniquités sexistes exige que nous prenions au sérieux la façon dont nous élevons nos garçons. Un investissement considérable dans les programmes sensibles aux spécificités sexuelles destinés aux garçons est un investissement dans la prochaine génération de maris, de pères et de citoyens.

\section{Références}

Archer, John. 1984. "Gender roles as developmental pathways." British Journal of Social Psychology 23: 245-256.

--- (éd.). 1994. Male Violence. London: Routledge.

Barker, Gary. 2000. "W hat about boys ? A review and analysis of international literature on the health and developmental needs of adolescent boys," O rganisation mondiale de la santé. <http://www.who. org> Consulté le 30 mars 2003.
- - - 2001. "Peace boys in a war zone: Identity and coping among adolescent men in a favela in Rio de Janeiro, Brazil." Erikson Institute, Loyola University, Chicago. Thèse de doctorat.

Gilmore, David. 1990. Manhood in the Making: Cultural Concepts of Masculinity. N ew Haven: Yale U niversity Press.

Mosher, D. et S. Tomkins. 1988. "Scripting the macho man: Hypermasculine socialization and enculturation," The Journal of Sex Research 25(1): 60-84.

O rganisation des Nations Unies. 1995. Rapport de la Conférence internationale sur la population et le développement. UND oc.A/CONF.171/13. N ew York: Organisation des Nations Unies. $<$ <ttp://www. unfpa.org> Consulté le 30 mars 2003.

Sonenstein, F., J. Pleck et L. Ku. 1995. Why Young M en Don't U se Condoms: Factors Related to the Consistency of U tilization. Washington, DC: U rban Institute. 


\section{«Mon père pensait autrement» \\ des garçons nigérians \\ réfléchissent à |'égalité \\ entre les sexes}

\section{par Françoise Girard}

En septembre 1994, Eddie Madunagu rentra chez lui à Calabar, dans l'État du Cross-River, dans la région sud-est du Nigéria. Madunagu, journaliste nigérian connu et respecté dans tout le pays, érudit et activiste politique, était à la croisée des chemins. Le bureau de rédaction de The Guardian, le journal progressiste où il travaillait à Lagos, venait d'être fermé par le régime militaire d'Abacha. Le mouvement socialiste nigérian auquel il appartenait, comme tous les autres mouvements socialistes après la chute de I'Union soviétique, était en proie au désarroi. Les convictions politiques de Madunagu

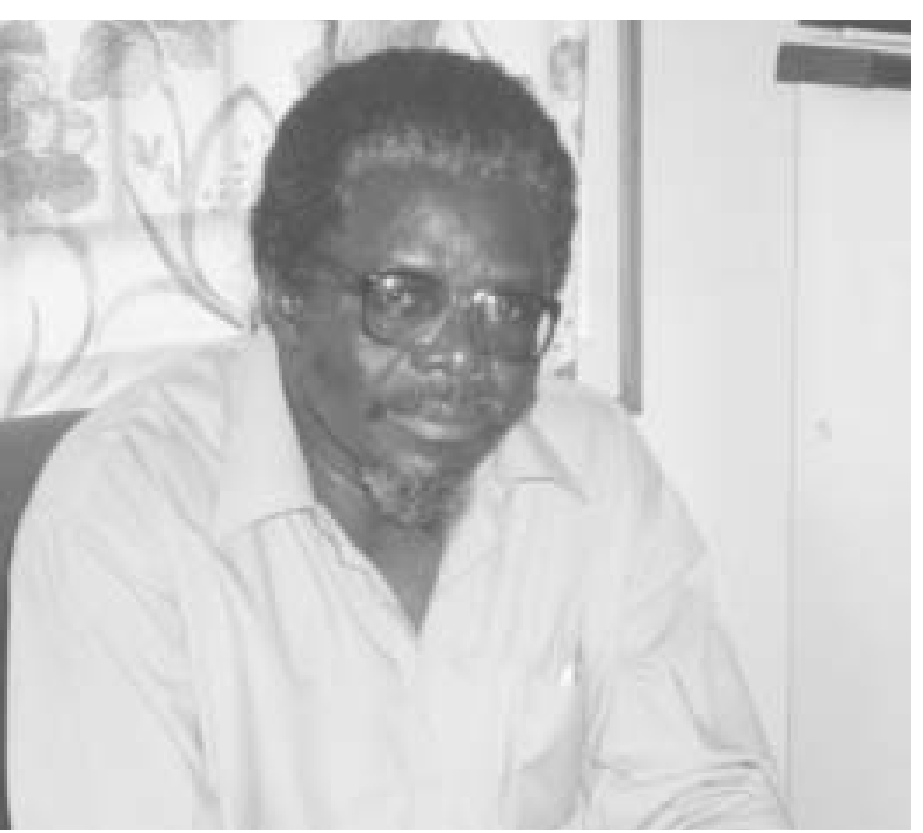

Eddie Madunagu est un homme chaleureux et charismatique dont la réputation d'intégrité absolue, l'énergie infatigable et la prodigieuse intelligence lui ont valu le respect et l'admiration de ses opposants politiques les plus tenaces.
I'empêchaient de retourner enseigner les mathématiques à I'U niversité de Calabar.

Après des années de lutte contre les dictatures militaires oppressives, y compris une période de détention dans sa jeunesse pour son radicalisme, Madunagu se sentait découragé par le manque de progrès du Nigéria depuis les jours exaltants de l'époque qui avait immédiatement suivi I'indépendance. Malgré la grande richesse en pétrole du pays, l'énorme majorité des N igérians sombrait dans une pauvreté toujours croissante. Les jeunes générations prenaient peu intérêt à l'engagement politique. L'injustice sociale et l'oppression s'observaient partout et la solidarité communautaire se désintégrait.

De retour à Calabar, Madunagu ne put rester oisif longtemps. A près avoir réfléchi quelque temps, il décida qu'il était temps de « revenir à l'essentiel » de son activisme progressiste-d'éduquer les jeunes gens, de les aider à développer un mode de pensée critique et de les conscientiser. Son premier geste fut de projeter l'ouverture d'une bibliothèque publique et d'un centre de documentation avec les nombreux livres qu'il avait collectionnés au fil des ans avec Bene, sa femme et co-activiste.

La pensée politique des Madunagu englobait les questions liées aux spécificités sexuelles (Voir « Garçons et filles du N igéria : qu'attendre de la vie ? » à la page 7). La conscience d'Eddie concernant les droits des femmes s'était au départ développée à partir de discussions avec Ingrid Essien-O bot, une féministe allemande marxiste qui enseignait à l'U niversité de Calabar jusqu'à son assassinat en 1981. L'engagement d'Eddie, en même temps 
que celui de Bene, en faveur de l'équité entre les sexes comme question de droits de la personne, ne fit qu'augmenter au cours des années ultérieures.

À Calabar, Bene venait de mettre sur pied l'initiative « Le pouvoir aux filles» (GPI, Girls' Power Initiative), une organisation qui encourageait l'autonomisation des adolescentes. Un soir, Andrea Irvin, I'administratrice de programmes pour l'Afrique de I'International Women's Health Coalition (IW HC), vint chez les Madunagu pour élaborer avec Bene le programme de G PI.

Eddie Madunagu se rappelle être entré et avoir dit en plaisantant : " Ne savezvous pas que les garçons ont eux aussi besoin de ce genre de programme ? Ils ne sont pas instruits. » En repensant à cette remarque, Madunagu explique qu'il s'inquiétait pour les filles de GPI qui, avec leurs connaissances nouvellement acquises, se retrouveraient face à des garçons « sans instruction » et des familles patriarcales. « Ça ne sert à rien de prier et de supplier un ignorant. ... . Les femmes ne peuvent pas se libérer en suppliant. » Madunagu pensait aussi au développement et à la croissance des garçons : " II est aussi nécessaire de faire savoir aux garçons qu'en fin de compte, les hommes ne peuvent pas l'emporter et qu'ils ne peuvent pas être vraiment heureux et libres

\section{Garçons et filles du Nigéria : qu'attendre de la vie?}

Le Nigéria, le pays le plus peuplé d'Afrique, a une population de 120 millions d'habitants dont la moitié est âgée de 19 ans ou moins. Au moins 20 pour cent de la population a entre 10 et 19 ans et 30 pour cent a moins de dix ans. L'avenir de ces jeunes gens sera déterminé par les troubles économiques et sociaux d'aujourd'hui, les pressions culturelles et parentales ainsi que les différences profondes dans les rôles et perspectives des adolescents et des adolescentes.

Les femmes et les filles sont considérées comme des subalternes et sont chargées de lourdes tâches à la maison. Beaucoup de familles n'assurent pas l'instruction de leurs filles parce qu'elles se marieront et rejoindront la famille de leur mari. La moyenne nationale d'âge au mariage est légèrement inférieure à 17 ans pour les filles, que l'on marie généralement à des hommes beaucoup plus âgés. Le mariage précoce signifie la fin des études et le début de nombreuses grossesses comportant chacune un risque élevé de complications et même de décès. Le Nigéria a l'un des taux de mortalité maternelle les plus élevés au monde.

Quoique beaucoup de filles grandissent dans des familles strictes qui les protègent jusqu'à ce qu'un mariage soit arrangé, d'autres sont confrontées à un harcèlement sexuel généralisé à l'école et ailleurs. À Lagos, par exemple, on envoie souvent les filles vendre des articles dans les rues où il se peut que des hommes les persuadent ou les forcent d'avoir des rapports sexuels. Les conséquences malheureuses sont évidentes : grossesses non désirées, avortements pratiqués dans de mauvaises conditions de sécurité, infections sexuellement transmissibles (IST) et taux d'infection à VIH croissants parmi les filles et les jeunes femmes. Lors d'une étude, 40 pour cent des filles du secondaire interrogées ont déclaré qu'elles avaient été enceintes au moins une fois.

Les garçons nigérians apprennent rapidement que leur société et culture considèrent les hommes comme supérieurs aux femmes_aussi bien dans le Nord musulman que dans le Sud chrétien. Les hommes prennent la plupart des décisions, y compris celles concernant les grossesses et les relations sexuelles. Les normes culturelles admettent les faibles taux d'emploi des préservatifs, les multiples partenaires sexuelles pour les hommes et la coercition sexuelle des filles. Le Nigéria est l'un des pays qui court le plus grave risque de voir le VIH/SIDA se propager à un rythme accéléré. Les adolescents, aussi bien les filles que les garçons, ont rarement accès à l'information et l'éducation de base concernant leur santé ou aux services de santé. 
tant qu'ils asservissent une partie de la société. . . . Ils doivent comprendre que leur supériorité et leurs avantages sont, en définitive, illusoires. . . . »

Irvin suggéra à Madunagu de créer un programme analogue à GPI pour les garçons. Bien qu'acquis à l'idée, Madunagu reconnaît volontiers avoir résisté pendant quelque temps. Finalement, déclare-t-il, «j'ai eu envie de développer davantage ma propre conscience des questions de genre. »C'est ainsi qu'est né un projet remarquable : "Conscientiser les adolescents de sexe masculin » (CMA, Conscientizing Male Adolescents).

\section{L'origine de CMA}

En décidant de travailler avec les garçons adolescents, Madunagu avait deux hypothèses sous-jacentes, expliquées dans les premiers documents de CMA :

Toutes les questions qui concernent les filles adolescentes, notamment la santé et les droits en matière de reproduction, la santé et les droits sexuels, les droits fondamentaux des personnes (y compris les droits spécifiques des femmes), l'ignorance, la pauvreté, l'impuissance et l'aliénation, l'exploitation et l'oppression, la violence et l'indignité concernent et doivent concerner les garçons adolescents et leurs aînés.

Eduquer les hommes sur les questions qui intéressent particulièrement les femmes est une contribution importante et même cruciale à la lutte universelle contre la discrimination et les injustices que subissent les femmes et qui sont perpétrées principalement par les hommes (ou dans leur intérêt), tant dans la vie privée que publique.

Irvin travailla avec M adunagu à l'élaboration d'une proposition pour un programme initialement conçu comme complément à GPI. Madunagu décida de travailler avec les garçons âgés de 14 à 20 ans parce qu'il estimait que les garçons de cette tranche d'âge étaient influençables et que typiquement, ils "s'imprègnent des préjugés sociaux les plus

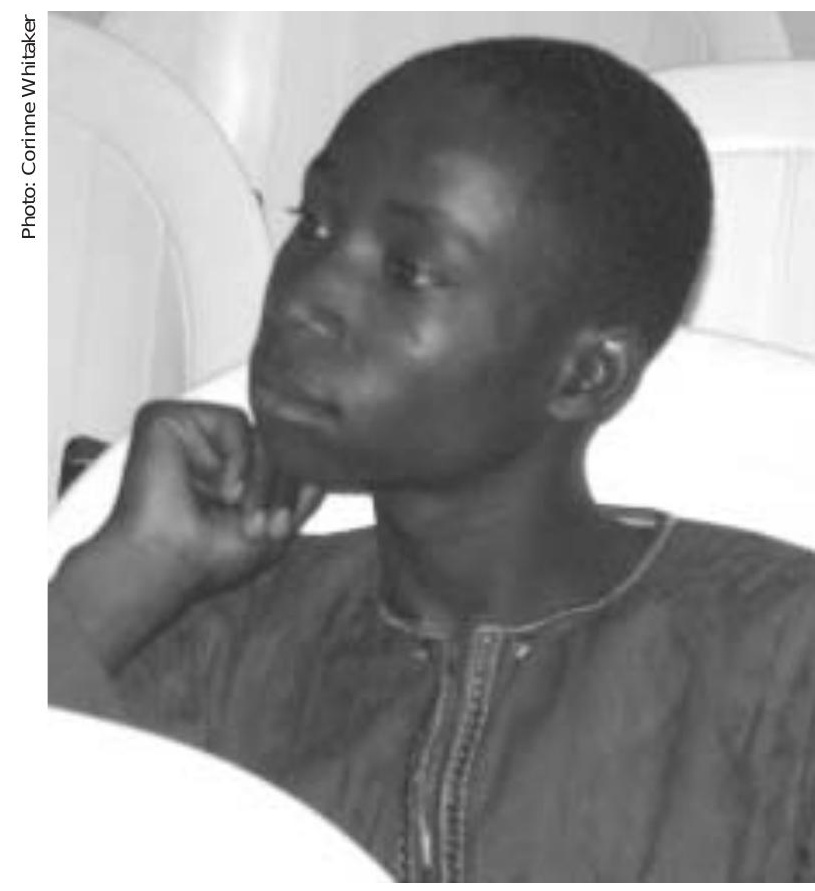

CMA a été fondé avec deux objectifs en tête : 1 ) sensibiliser les garçons à l'oppression sexiste et 2) développer leur aptitude à la pensée critique en leur apprenant à analyser le monde qui les entoure afin d'établir eux-mêmes un nouvel ensemble de valeurs.

arriérés contre les femmes. » II était en outre convaincu que les garçons étant les «bénéficiaires » d'un système caractérisé par l'inégalité et l'oppression, il ne serait pas possible de les atteindre de manière efficace avant l'âge d'au moins 14 ou 15 ans, âge auquel ils deviennent capables de participer à un débat intellectuel et d'établir des parallèles entre le patriarcat et les autres formes de pouvoir oppressif, comme l'oppression des minorités ethniques et l'exploitation des pauvres.

Le principal objectif du programme CMA allait être de faire participer continûment les adolescents à des groupes de discussion afin de mieux les sensibiliser à I'oppression sexiste. Un deuxième objectif, tout aussi important, serait de développer l'aptitude des participants à la pensée critique et à l'analyse, de leur apprendre « à remettre en question l'information et la réalité avant de s'incliner devant elles», comme le dit Madunagu. De cette façon, les garçons pourraient par euxmêmes parvenir à un nouvel ensemble de 
valeurs, intérioriser ces valeurs et aiguiser leur capacité d'analyser ce qu'ils percevaient du monde qui les entourait. En plus des groupes de discussion, CMA offrirait des services de conseils socio-psychologiques et mènerait des actions de sensibilisation communautaires.

Madunagu parvint à mettre sur pied une organisation formelle-I'Institut international de Calabar pour la recherche, l'information et le développement (CIIN STRID, Calabar International Institute for Research, Information and Development) - a fin de fournir une affiliation institutionnelle au CMA et abriter la bibliothèque et le centre de documentation (voir cidessous l'information sur le CIIN STRID).

En juin 1995, Madunagu parvint à recruter auprès de trois écoles secondaires un groupe-pilote de 25 adolescents qui désiraient participer à des séances de discussion hebdomadaire de deux heures pendant une année entière. Ces garçons étaient en majorité des élèves talentueux et des leaders sociaux. Madunagu se servit des discussions pour identifier les sujets de discussion les plus pertinents et élaborer un programme soutenu et intensif. Après l'année pilote, le programme se développa rapidement et les premières

\section{L'Institut international de Calabar pour Ia recherche, I'information et le développement (CIINSTRID, Calabar International Institute for Research, Information and Development)}

Le CIINSTRID, l'institution dont dépend le projet « Conscientiser les adolescents de sexe masculin » (CMA, Conscientizing Male A dolescents), abrite aussi une bibliothèque de recherche et un centre de documentation. En activité depuis 1996, le centre est ouvert gratuitement à tous les membres de la communauté sept jours sur sept. Située au premier étage d'un modeste immeuble, sa bibliothèque abrite une impressionnante collection de livres, de journaux et de revues sur des sujets comme l'histoire et la politique du Nigéria, le marxisme, la planification des carrières professionnelles, la religion, le féminisme et la santé sexuelle et reproductive. Au cours des dernières années, la collection a été enrichie par des dons de plusieurs éminents écrivains et académiciens nigérians, ainsi que de l'International Women's Health Coalition (IWHC). Aujourd'hui, les livres et les revues envahissent presque tous les locaux de CIINSTRID. Jeunes et vieux des deux sexes fréquentent assidûment la bibliothèque, et viennent consulter des documents n'existant nulle part ailleurs à Calabar ou même au Nigéria. La bibliothèque est un pôle d'attraction pour les jeunes en particulier.

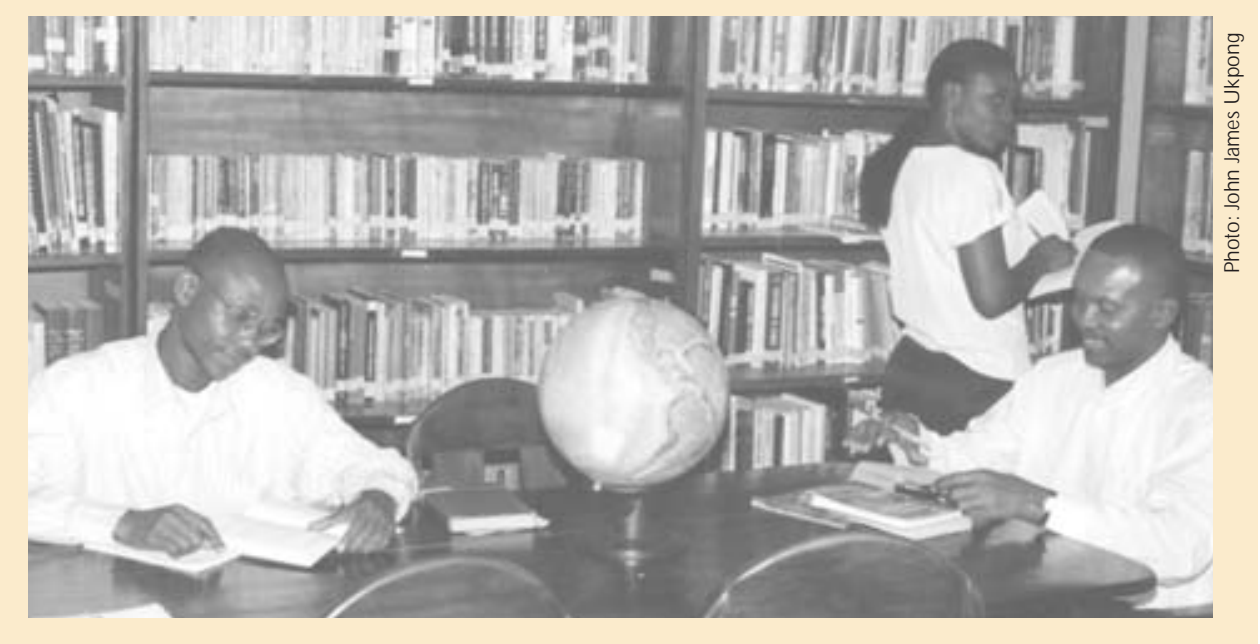


années nécessitèrent une restructuration constante. CMA commença à organiser les séances dans les écoles locales, puis (avec un financement supplémentaire de la Fondation MacArthur) le projet s'étendit à des écoles d'U yo, une ville de l'État voisin d'Akwa-lbom. Pour prendre en charge l'effectif croissant (250 garçons en 1997), Madunagu commença à recruter et former des enseignants pour diriger les groupes. ${ }^{1}$

La croissance et le développement rapides de CMA créèrent plusieurs dilemmes pendant les premières années. Premièrement, la formation des enseignants du secondaire pour mener les discussions devint bientôt un défi accablant. D euxièmement, beaucoup de nouveaux professeurs trouvèrent difficile de couvrir le programme intensif en un an. Troisièmement, à la fin du cours, beaucoup de garçons voulaient continuer à se réunir pour apprendre.

En 1997, Madunagu décida de modifier le programme de CMA en réduisant le programme initial, mais en ajoutant une deuxième année pour les garçons les plus prometteurs et les plus engagés. Ces pro-

\section{Bien que Madunagu ait initialement}

orienté le programme vers des

questions sociales d'ordre général,

il a vite appris que les garçons

s'intéressaient aussi à l'amour, aux

relations familiales et à la violence

domestique.

grammes consécutifs furent appelés $\mathrm{N}$ iveau I et N iveau II. Le N iveau I consiste toujours en des réunions hebdomadaires de deux heures dans diverses écoles. Les groupes du Niveau II tiennent des réunions mensuelles d'une journée dans un hôtel de Calabar. Ils comprennent 45 participants âgés de 17 à 19 ans provenant de divers groupes du Niveau I.

\section{Le contenu du progiramme}

Du fait des propres tendances intellectuelles de Madunagu, le programme initial de CMA était axé sur I'histoire nigériane et sur une analyse sociale générale de l'oppression sexiste. Toutefois, l'expérience initiale montra que les garçons s'intéressaient aussi aux sujets qui les concernaient personnellement, comme l'inégalité dans la famille, la violence contre les filles et les femmes ainsi que les relations entre les garçons et les filles. Madunagu en conclut que l'intégration d'approches psychosociales à la matière, comme l'avait suggéré Irvin lors de leurs premières discussions, permettrait d'atteindre plus efficacement les garçons.

Actuellement, le programme du Niveau I traite des concepts fondamentaux de genre et de société, en commençant par une unité sur les spécificités sexuelles et le sexisme comme constructions sociales. D ans cette unité, les élèves comparent les différences biologiques entre les hommes et les femmes avec les différences dans leurs rôles sociaux et leur pouvoir dans la société nigériane. Ils se rendent progressivement compte que la biologie seule ne peut pas expliquer l'inégalité entre les sexes et qu'une telle inégalité est injuste. La leçon suivante permet de comprendre comment cette injustice est perpétuée dans la famille traditionnelle, considérée comme une « sphère privée » dans la culture nigériane. Les participants explorent et débattent le fait que les femmes et les filles-leurs mères et leurs sœurs, par exemple-sont souvent opprimées dans la famille et que les adolescents et les enfants des deux sexes peuvent aussi être soumis au mauvais traitement ainsi qu'à la violence dans la famille. À mesure que leur conscience et leur compréhension augmentent, les participants examinent la violence contre les femmes comme phénomène personnel et social. Une attention particulière est

1 À mesure que l'organisation s'esta agrandie, le rôle de Madunagu a également changé. En général, il ne dirige plus que la visite annuelle des élèves au CIIN STRID. 


\section{Programme actuel de CMA}

\section{Niveau |}

- Sexisme, biologie et conscience

- Discrimination à l'égard des femmes et des enfants dans la famille et la société

- Violence contre les femmes et responsabilité des hommes, de l'État et des autorités traditionnelles : le viol, la mutilation sexuelle, le harcèlement sexuel

- Santé et droits sexuels et reproductifs

- Entraves culturelles à l'égalité entre les sexes

- Responsabilité des hommes dans les relations sexuelles, l'amour et le mariage

- Écologie humaine et reproduction (conditions sociales et physiques pour une reproduction saine)

\section{Sujets supplémentaires pour Ie Niveau II}

- Compétences en communication et usage de l'anglais

- Logique et méthode mathématique

- Introduction critique et anti-sexiste à la société nigériane

- Introduction critique et anti-sexisteà I'histoire du monde

- Introduction critique et anti-sexiste à la démocratie et aux droits de la personne

- Le féminisme et le mouvement des femmes

- Conscientisation et société (critique du présent et vision du futur)

- Conscientisation pratique (intervention sociale et éducation par les pairs)

- Adolescents et société

accordée à la violence parce qu'elle est très répandue dans la société nigériane. Bien que le programme aborde des sujets de santé sexuelle «traditionnels » comme les préservatifs, la contraception et les IST/NIH, une moindre importance leur est accordée.

L'élaboration des programmes est un processus continu. II y a quelques années, quand les garçons avaient manifestement du mal à distinguer l'amour du désir sexuel, une unité traitant de « la responsabilité des hommes dans les relations sexuelles, l'amour et le mariage » a été ajoutée au programme du Niveau I. L'unité était adaptée d'une leçon de GPI.

Le cours du Niveau II est une révision des sujets abordés au Niveau I, mais il est beaucoup plus axé sur le développement des compétences des garçons en matière de communication effective, d'abstraction et d'analyse critique. Le programme inclut des sujets comme la logique, et un cours de rattrapage d'anglais pour appuyer ce renforcement des capacités. Une autre façon d'aider les élèves à comprendre l'oppression sexiste consiste pour les animateurs à faire des analogies avec d'autres exemples d'oppression, comme la pauvreté ou la violence ethnique. Le cours du Niveau II développe aussi les capacités pratiques des garçons afin qu'ils puissent intervenir dans leur communauté et auprès de leurs pairs, par exemple lors d'incidents de harcèlement sexuel. L'analyse des spécificités sexuelles se focalise toujours essentiellement sur l'expérience des femmes, mais $M$ adunagu est en train d'élaborer des matériels pédagogiques concernant l'effet négatif sur les hommes de la masculinité telle qu'elle est traditionnellement définie. II cherche des moyens d'aborder la masculinité de manière "pas trop abstraite », par exemple en introduisant des discussions sur les sentiments qu'éprouvent les garçons quand ils ne sont pas bons en sport, ou quand leurs pairs masculins font pression sur eux pour qu'ils pourchassent les filles ou qu'ils soient «macho » en affichant prouesses physiques et agressivité.

Le paradigme de CMA est différent de celui de la plupart des programmes visant les adolescents. Les programmes plus conventionnels destinés aux garçons mettent l'accent sur la santé sexuelle, en particulier l'éducation sur les préservatifs et 
la distribution de ceux-ci, et ils sont souvent organisés dans des complexes sportifs et des maisons de jeunes. Les contacts « éducatifs » avec les jeunes peuvent être limités à quelques séances. Le programme de CMA, par contre, est distinct :

1. L'accent est mis principalement sur le sexisme et la pensée critique et non sur la prévention des grossesses non désirées, des IST et du VIH.

2. Le pouvoir et l'oppression sont discutés en profondeur.

3. Le temps de contact minimum est d'un an, et hebdomadaire.

4. Le programme touche non pas les garçons les plus « à risque », mais certains des garçons les plus intelligents et les plus motivés, qui sont des leaders dans leurs établissements.

5. Les activités supplémentaires sont essentiellement académiques plutôt que sociales ou athlétiques.

6. Le programme est étroitement lié à un projet parallèle destiné aux filles, GPI, qui fournit une assistance technique.

Un tel programme pourrait ne pas sembler très intéressant pour des adolescents, et peut-être que les jeunes hommes intelligents et pleins d'assurance que CMA retient s'auto-sélectionnent dans une certaine mesure. Q uand j'ai demandé à des garçons de CMA pourquoi ils s'étaient inscrits au programme, les raisons les plus fréquemment invoquées étaient qu'ils aiment fréquenter les garçons de leur âge, qu'ils éprouvent de la curiosité au sujet des buts du programme, notamment lutter contre l'oppression et le sexisme, et qu'ils veulent apprendre à penser de façon critique et comprendre le monde dans lequel ils vivent. Contrairement aux attentes, aucun d'entre eux n'a mentionné ni l'inscription de ses amis, ni l'occasion de recevoir des cours particuliers (de maths et d'anglais), ni la réputation de M adunagu. En effet, la plupart des garçons n'avaient jamais entendu parler de Madunagu avant de s'inscrire au programme.

\section{Une approche basée sur le dilalogue}

Les groupes de discussion de CMA utilisent une méthode dialogique modifiée inspirée de la Pédagogie des Opprimés (1970) de Paolo Freire. Cette méthode associe le dialogue et l'argument logique à des informations correctes, souvent techniques. Les garçons peuvent vérifier euxmêmes les faits qui leur sont présentésdes exemples tirés de la vie dans leur communauté et au N igéria.

Au début de chaque séance, l'animateur présente un sujet et donne quelques définitions. Au lieu du processus traditionnel d'apprentissage par cœur, les garçons sont amenés à percevoir et analyser les contradictions de leurs propres opinions grâce à une série de questions et de réponses. Le but est de pousser le participant à dégager lui-même les conclusions. Si ce n'est pas possible, I'animateur vise un objectif plus restreint, à savoir, faire admettre à un garçon qu'une contradiction est survenue entre ses croyances et les faits qu'il reconnaît comme étant vrais. Après avoir fait participer les élèves au dialogue, l'animateur résume la discussion et propose d'autres déductions qui pourraient ne pas avoir surgi lors de la discussion.

$\mathrm{Au}$ cours des dernières années, à la suite de la formation du personnel dispensée par GPI, CMA a aussi adopté plusieurs autres méthodes et techniques d'apprentissage comme les jeux de rôle, le remueméninges, les exercices « vrai ou faux » et les « mythes et réalités ». L'association de ces multiples techniques aux discussions s'est avérée efficace et intéressante pour les garçons du secondaire.

Q uand j'ai visité le CIINSTRID et CMA en février 2002, à la fin de la septième année du projet, je me posais beaucoup de questions. Comment CMA pouvait-il convaincre des adolescents qu'ils étaient des oppresseurs et qu'ils devaient changer ? Les garçons changeraient-ils vraiment ou se contenteraient-ils de parler sans réelle conviction de l'égalité entre les sexes?

J'ai assisté à la cérémonie de remise des diplômes aux participants à CMA de Hope Waddell Training Institute et de 
West African Peoples' Institute, deux établissements d'enseignement secondaire de Calabar où le programme CMA est actif. Le programme se composait d'une discussion dialogique sur le viol et d'un sketch sur le mariage forcé et la dot. L'approche s'avéra intéressante.

\section{Méthode dialogique : réflexion sur le viol}

Igwe Dermot, directeur adjoint de Hope Waddell et coordonnateur des responsables de terrain de CMA, engagea une discussion animée. U tilisant un tableau de conférence et des marqueurs, il demanda aux garçons quels mots doux ils disent à leurs petites amies quand ils se trouvent dans une situation intime. Au milieu des fous rires, des réponses fusèrent de toutes les directions :

«Chérie, que tu es adorable!»

«Mon amour, je t'aime plus que ma mère. »

«Ma chérie, rends-moi fou !»

«Tu es le sucre dans mon thé. . . . »

Dermot demanda ensuite aux garçons ce que leurs amies leur disent à ces moments-là. Les garçons levèrent la main avec enthousiasme :

«Chéri, je t’aimerai toujours. »

«Tu me tues à petit feu. »

« S'il te plaît, tiens-moi et ne me lâche

pas. »

« Vas-y doucement, je meurs. »

Les garçons durent ensuite comparer ces mots avec ceux que les filles leur disent quand elles ne veulent pas avoir de rapports sexuels ou passer du temps avec eux :

«Laisse-moi, s'il te plaît. »

« Je ne me sens pas bien. »

« Je n'ai pas envie. »

«Tu es complètement fou. »

"L'amour n'a rien à voir avec les rapports sexuels. »

Se servant des réponses, Dermot souleva une discussion sur les raisons qui font penser aux garçons qu'une fille veut en fait dire « oui » lorsqu'elle dit « non ». U ne repartie énergique s'ensuivit :

«Les filles ne se voient pas du tout disant oui, et c'est pour cela qu'elles disent toujours non. »

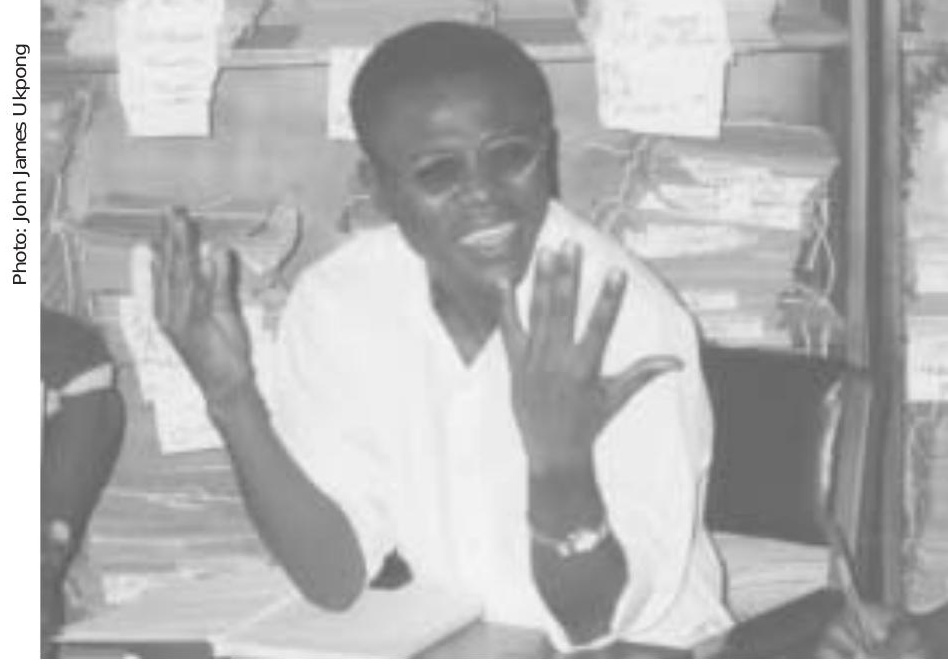

Les responsables de terrain concluent les discussions sur le viol en encourageant les garçons à faire un remue-méninges sur les moyens d'aborder la violence sexuelle.

«Ce n'est pas vrai, une fille peut bien dire oui. Ça, je le sais.» «Si elle vient chez moi, cela ne veut-il pas dire qu'elle est prête à tout ? »

Dermot pose en retour la question suivante : "Ma voisine vient chez moi pour me parler, cela veut-il dire qu'elle est prête à tout ? »Certains garçons répondent par la négative. Un sceptique, Stanley, dit soudain : «M Mais les actes sont plus éloquents que les paroles. »

Dermot : «Q uels sont ces actes ?»

Stanley : « Le fait de porter une minijupe. . . de s'asseoir près de moi. ... »

Une animatrice âgée de 32 ans intervient : "Mais je suis assise près de ce garçon! » (Le groupe rit.)

Stanley : «Et la mini-jupe ?»

Dermot: «Vous connaissez les fous et les folles qui se promènent tout nus sur la grand-route, n'est-ce pas ? Cela veutil dire qu'ils sont prêts pour les rapports sexuels?»

Stanley : «Non! »

Dermot: «Et quand je m'asseois dans ma salle de séjour en caleçon, avec ma chemise ouverte, cela signifie-t-il que je suis prêt pour les rapports sexuels? »

Le groupe : «Non! »

Stanley : " J'ai bien peur que vous ne soyez en train de dire que nous ne pouvons pas avoir de rapports sexuels. »

Un membre du personnel de CMA : "Mais si, vous pouvez avoir des rapports sexuels si vous le voulez et si la fille le veut. Mais la question est de savoir si la fille le veut. » 
Stanley se cale dans son siège, retournant les réponses dans sa tête. Finalement la majorité des garçons, reconnaissant qu'ils veulent bien dire non quand ils disent non, concluent qu'il en est de même pour les filles.

La discussion se focalisa ensuite sur la définition du viol. Un débat s'ensuivit pour déterminer si le viol est préférable à la masturbation. Le groupe en conclut que juxtaposer ainsi la masturbation et le viol n'était pas approprié et que le viol est toujours inadmissible. Certains garçons dirent avoir besoin de plus d'information sur la masturbation. Le sujet suivant fut de savoir si une femme peut violer un homme, un sujet manifestement fascinant aux yeux des participants! En dernière analyse, les garçons s'accordèrent pour dire que le viol des hommes par les femmes n'est pas un problème social important au N igéria.

Les garçons discutèrent ensuite des raisons du viol en examinant les relations de pouvoir entre les hommes et les femmes ainsi que les croyances selon lesquelles les hommes doivent dominer et avoir de l'autorité sur les femmes. La séance se termina par un remue-méninges pour déterminer ce que les garçons pou- vaient faire en tant qu'activistes pour résoudre le problème du viol. Ils décidèrent qu'ils pouvaient informer les autres hommes et garçons sur le viol, les rendre conscients que le viol c'est de la violence, conseiller les femmes et les filles sur la prévention du viol et quoi faire en cas de viol, accompagner les filles ou les femmes violées au commissariat de police ou à I'hôpital, intervenir dans les cas de violence, s'élever contre le harcèlement des filles et changer les lois nigérianes afin de reconnaitre le viol conjugal comme une forme de viol.

Tout au long de la discussion, il était évident que tous les garçons n'avaient pas le même niveau de conscience. Toutefois, le climat de dialogue permettait à tous de s'exprimer franchement. Un bon nombre de garçons, qui avaient manifestement compris et intégré une critique du sexisme dans leur pensée, étaient capables de présenter des arguments d'une manière pertinente et persuasive. Quelques-uns d'entre eux continuaient néanmoins de s'opposer à certaines idées, bien que c'était le jour de la remise des diplômes et qu'ils étaient en présence d'un observateur extérieur. D'autres demandèrent de plus amples informa-

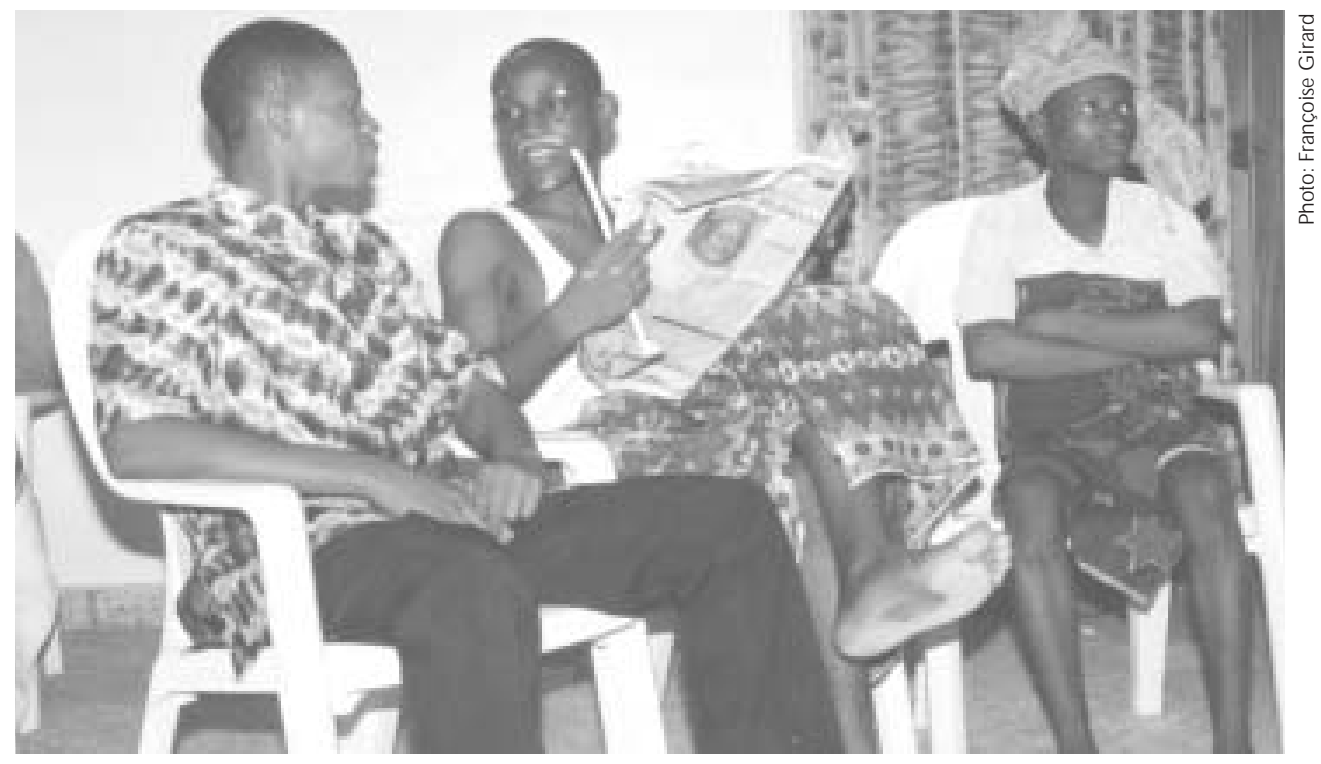

Dans un sketch sur les traditions de mariage, les garçons critiquèrent certains aspects de la culture nigériane traditionnelle, comme la dot, tout en montrant une grande prédilection pour beaucoup d'autres aspects de leur culture, comme son trésor de proverbes. 
tions sur certains sujets. Certains membres du personnel de CMA même semblaient encore se débattre avec certains sujets. Par exemple, I'un d'entre eux, qui avait récemment achevé sa formation de $\mathrm{N}$ iveau II, écarta «le problème du lesbianisme [comme] n'étant pas si marqué au Nigéria », et personne ne contesta sa déclaration.

Dans l'ensemble, la discussion fut remarquablement franche. Les garçons n'avaient pas peur d'utiliser la terminologie appropriée, par exemple pénis, érection, désir sexuel et relations sexuelles. Le langage de la moralisation et de la honte était absent. Dermot était un animateur talentueux, qui savait rendre la discussion vivante et même amusante. L'atmosphère et le ton étaient positifs et respectueux, et la majorité des garçons participèrent. La deuxième discussion sur le même sujet, que j'observai quelques jours plus tard à U yo lors de la cérémonie de remise des diplômes pour le Niveau I, fut similaire.

\section{Sketch : Le mariage forcé et la dot}

Le sketch sur le mariage forcé et la dot, qui suivit la discussion, était à la fois désopilant et poignant. En costume et maquillés, les garçons jouèrent l'histoire d'une fille qui ne peut pas épouser le garçon du village qu'elle aime parce que son père exige une dot élevée du futur marié. Elle est contrainte d'épouser un homme plus âgé de Lagos qui a les moyens de payer. Son mariage est cruellement malheureux et sa situation s'empire avec la mort de son mari. Les anciens du village entrent en scène et prétendent qu'elle doit être pour quelque chose dans la mort de son mari. Ils veulent la forcer à boire l'eau qui a servi à laver le cadavre « pour prouver qu'elle a les mains propres ». Dans une discussion ultérieure, les garçons exprimèrent leur sentiment d'injustice à l'idée de ne pouvoir se marier par amour parce qu'on n'a pas les moyens de payer.

\section{Formation des responsables de terrain et du personnel}

Pour mener les discussions du Niveau I, les dirigeants de CMA recrutent et forment un ensemble de " responsables de terrain », qui sont enseignants dans les écoles participantes. ${ }^{2}$ Comme I'explique Paul Awah, un diplômé de CMA et animateur adjoint âgé de 21 ans, l'identification de responsables de terrain potentiels est un défi permanent. II est difficile de trouver des hommes adultes qui veulent étudier le sexisme, sont réceptifs aux idées nouvelles et peuvent enseigner ou guider.

Au départ, Madunagu fit un «saut dans l'inconnu », comme il le dit lui-même, en demandant aux directeurs des écoles de choisir les enseignants à former. Toutefois, les directeurs suggéraient souvent des candidats inadéquats : certains n'étaient intéressés que par la compensation financière, d'autres ne pouvaient pas maîtriser la méthode dialogique ou modifier leurs attitudes invétérées à l'égard des femmes. Plus récemment, la sélection des enseignants semble bien se dérouler ; les membres du personnel de CMA, les responsables de terrain actuels, les diplômés de CMA et les participants actuels y contribuent tous.

La formation des responsables de terrain a lieu à CM $A$ et comprend une séance intensive de cinq jours, suivie de quatre séances mensuelles d'un jour. À cause du temps et des dépenses qu'entraine le déplacement quotidien de Calabar à U yo, les dirigeants de CMA pensent à l'avenir héberger les enseignants d'U yo à Calabar pendant la formation et mener celle-ci en deux séances intensives de cinq jours (une en hiver et une en été), avec des réunions de révision en automne et au printemps.

Depuis un certain temps, Madunagu avait conclu qu'au cours de la formation, CMA n'accordait pas à certains sujets l'importance qui leur était due. II décida

\footnotetext{
2 CMA dispense aussi une formation de base danssur la plupart des aspects du programme à tout le personnel, y compris le personnel administratif qui ne dirige pas les groupes de discussion.
} 


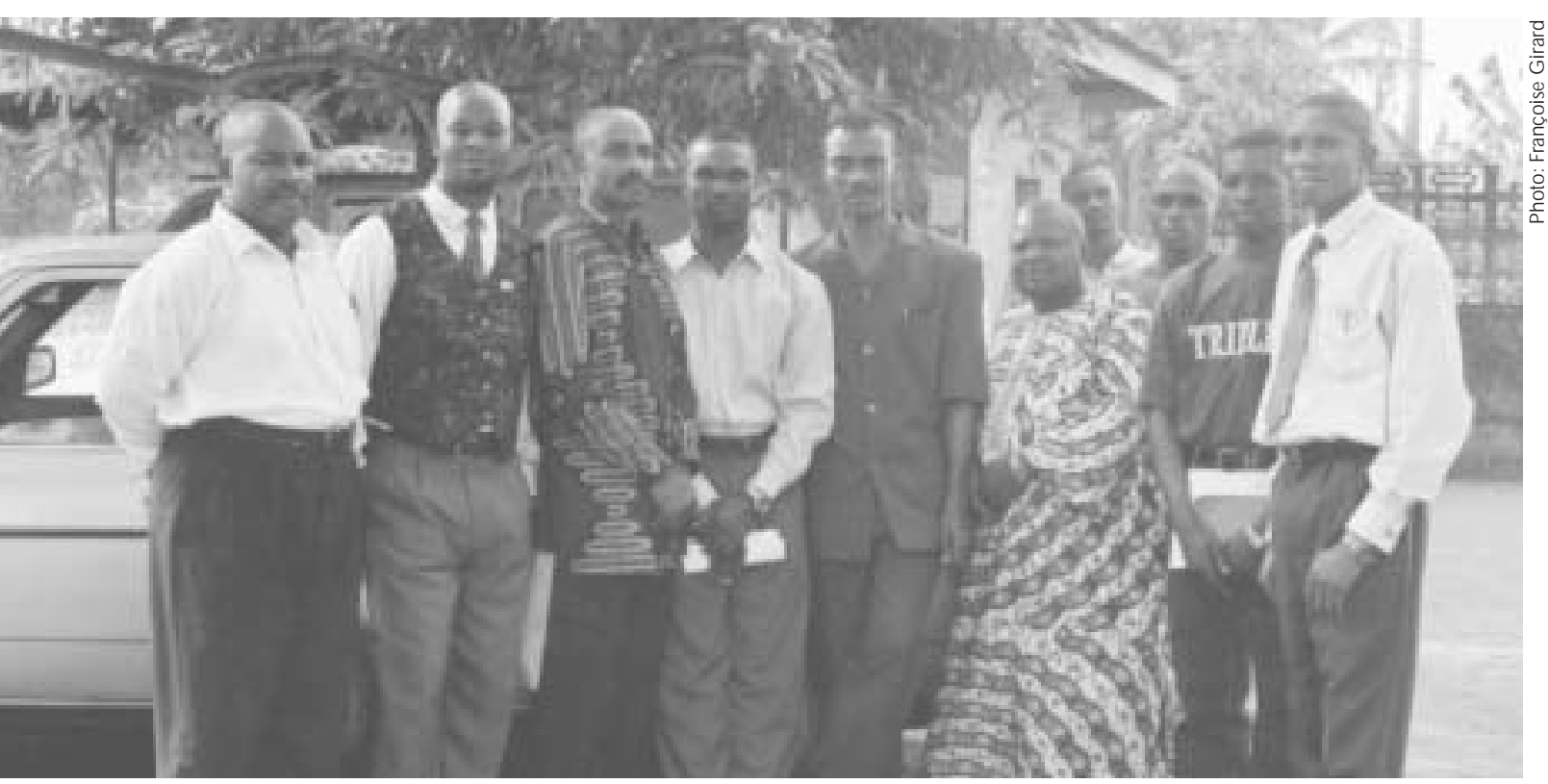

En identifiant les potentiels responsables de terrain, les dirigeants de CMA cherchent des hommes adultes qui veulent étudier le sexisme, sont réceptifs aux idées nouvelles et peuvent enseigner ou guider. Le défi est de taille.

donc de renforcer les relations entre CM A et GPI. Le personnel de GPI est en général plus expérimenté que celui de CMA, et il a reçu une formation plus spécialisée au Nigéria et à l'étranger. Depuis I'an 2000, le personnel de GPI assure les séances de formation pour les thèmes du Niveau I, notamment les responsabilités des hommes, le féminisme ainsi que la santé et les droits sexuels et en matière de reproduction. Les formateurs de CMA continuent à traiter des sujets du Niveau II tels que les compétences en communication, I'anglais et I'histoire du N igéria.

Les discussions du Niveau II sont menées par les membres du personnel de CMA, dont certains sont des adolescents diplômés d'un cours de Niveau II antérieur. La formation des animateurs des groupes de Niveau II est assurée par des membres du personnel de CMA plus expérimentés ainsi que par le personnel de GPI. Elle met un accent particulier sur les capacités d'expression orale et les " techniques de conscientisation », I'art de mener les débats dialogiques, et autres exercices en groupes.

En l'an 2000, les dirigeants de CM A ont élaboré un manuel basé sur des notes qu'ils avaient compilées pendant plusieurs années, afin de guider les responsables de terrain et le personnel lors des discussions de groupe. Le manuel précise les objectifs et expose brièvement les grandes lignes de chaque séance. II propose aussi des questions et des activités et inclut une courte liste d'ouvrages de référence pour lecture supplémentaire. Cette information constitue un bon point de départ, mais la plupart des responsables de terrain ont besoin de conseils supplémentaires, par exemple une description pas à pas des exercices. En conséquence, les dirigeants de CMA ont produit des notes de discussion plus détaillées sur chaque thème. Le manuel sera bientôt développé et révisé, avec l'aide du personnel de GPI.

\section{Au-delà du Niveau ||}

Il y a quelques années, un petit groupe de diplômés du Niveau II ont formé l'« avantgarde de CMA » (CMA Vanguard) pour s'exprimer et organiser des discussions et activités dans leurs communautés. Bien que ces jeunes hommes soient particulièrement intelligents et engagés, ce sont encore des adolescents qui ont du mal à agir systématiquement sans le soutien continu de 
CMA. En conséquence, ils sont en train d'effectuer une transition à un groupe de « Niveau III » qui se réunit régulièrement avec le personnel de CMA. Les diplômés des Niveaux II et III sont, à bien des égards, les cadres d'une nouvelle génération de jeunes leaders dans leurs communautés.

À mesure que les membres du personnel de CMA devinrent plus expérimentés dans le travail avec les adolescents plus âgés, ils commencèrent à travailler avec un groupe d'étudiants de I'U niversité de
Calabar âgés de 18 à 25 ans. Un bon nombre des participants ne connaissant pas CMA (n'ayant pas suivi les N iveaux I à III), cette composante est appelée « Groupe spécial ». Depuis 1999, les 25 étudiants du Groupe spécial, encadrés par le personnel de CMA, se réunissent une fois par semaine pendant deux heures. Ils se concentrent sur la violence à l'égard des femmes et le harcèlement sexuel, qui sont des problèmes particulièrement graves à l'université.

\section{Études de cas relatives aux conseils socio-psychologiques*}

\section{A deyen i, 16 ans, Niveau ||}

Adeyeni a vu un homme battre une femme et déchirer ses habits. Bien que ne connaissant pas ces deux personnes, il pense qu'il aurait dû faire quelque chose pour mettre fin à la raclée. II veut savoir ce qu'il aurait pu faire. En se fondant sur leur discussion, le conseiller convient avec lui que, vu les faits particuliers de cette situation, il n'aurait probablement pu rien faire directement, sauf appeler la police.

\section{Stanley, 19 ans, N iveau I}

Stanley est follement amoureux d'une fille qui était séparée de son premier copain quand elle l'a rencontré. Peu après, elle s'est réconciliée avec son premier copain et a abandonné Stanley. Stanley déclare qu'en ce moment il n'arrive ni à manger, ni à se concentrer, ni même à jouer au volley. Le conseiller et lui parlent de la courte relation qu'il a eue avec cette fille. Stanley conclut que lui seul était amoureux et que la fille s'est servie de lui comme pont émotionnel pendant sa séparation avec son premier copain. II dit qu'il sortira beaucoup plus pour voir des amis afin d'oublier la fille. II ajoute qu'il est triste, mais qu'il n'est plus tendu.

\section{Duke, 17 ans, Niveau II}

Duke est réservé et a du mal à communiquer avec les gens. Il aimerait partager les connaissances qu'il a acquises à CMA, mais il ne peut pas se résoudre à le faire. La conseillère se rend compte qu'il n'est pas aussi timide avec ses frères et sœurs. Elle suggère donc que dans un premier temps, il s'entraîne à leur parler de CMA. Elle lui suggère aussi de demander à l'un de ses frères de l'accompagner quand il voudra parler à d'autres personnes de CMA.

\section{B enjamin, 18 ans, Niveau II}

Benjamin veut s'abstenir totalement jusqu'au mariage à cause de ses croyances religieuses, mais il n'est pas sûr de pouvoir y parvenir. Le conseiller le sonde pour savoir ce qu'il entend par abstinence totale. Ils discutent des différentes manières d'exprimer l'amour et les sentiments sexuels sans avoir de rapports sexuels complets. Bien que Benjamin dise qu'il ne peut pas considérer la masturbation à cause de sa religion, il pense qu'il peut envisager les méthodes sans pénétration pour extérioriser sa sexualité.

* Tous les noms ont été changés pour protéger la vie privée des élèves. 


\section{Le service de conseils socío-psy chologi ques}

Peu après l'établissement du programme, Madunagu et son personnel se sont rendu compte que plusieurs garçons avaient besoin de conseils personnalisés. Certains avaient des problèmes personnels tandis que d'autres voulaient parler de leurs aspirations et buts personnels ou poser plus de questions sur les sujets suscités au cours des réunions de groupe. Un service, initialement plus apparenté à l'éducation qu'aux conseils intensifs en tête-à-tête, a été établi en 1996. En 1999, les dirigeants de CMA ont révisé l'approche et engagé un professionnel pour aider les garçons en matière de résolution de problèmes et de prise de décision. Malheureusement, le travail du premier conseiller s'est avéré insatisfaisant et le service était peu utilisé. En 2001, les dirigeants de CMA ont contracté les services des conseillers de GPI,

\section{CMA continue à s'appuyer sur la} Girls' Power Initiative pour

certains aspects de sa formation

des enseignants ainsi que pour

pourvoir en personnel le service

de conseils socio-psychologiques.

qui passent maintenant jusqu'à cinq heures par semaine à CMA. Pendant les premiers mois de ce service amélioré, I'utilisation est passée à une moyenne de sept élèves par semaine. Néanmoins, le service demeure sous-utilisé en partie à cause des entraves culturelles ( « c'est une idée étrangère ») et aussi parce que les responsables de terrain n'ont pas attiré l'attention de leurs élèves sur le service ou ne les y ont pas adressés. Les dirigeants de CMA sont en train d'encourager activement les responsables de terrain à orienter les élèves vers ce service.

Tous les participants à CM A sont censés participer une fois par an à une séance de conseils personnalisés, et la plupart d'entre eux le font. Cette séance donne aux garçons et au personnel de CMA I'occasion de discuter de tout sujet de préoccupation avec un conseiller, qu'il s'agisse d'un problème familial ou d'une question en rapport avec l'école ou une carrière. En outre, les garçons peuvent venir pour des conseils particuliers au besoin. Les garçons à qui j'ai parlé, et qui avaient choisi de voir le conseiller, ont déclaré qu'ils étaient satisfaits des conseils et de l'information reçus, à l'exception d'un étudiant du $G$ roupe spécial qui pensait que les conseils qu'il avait reçus étaient plus appropriés à des garçons plus jeunes. Un garçon a indiqué qu'il était reconnaissant de la confidentialité des conseils à CMA, ce qui ne caractérisait pas les autres services similaires offerts aux jeunes gens à Calabar.

\section{Le travail}

\section{communautailre}

Beaucoup de diplômés de CMA sont formés à intervenir dans leur communauté ainsi qu'auprès de leurs pairs quand ils assistent à des incidents qui violent les droits des femmes. Les compétences activistes sont initialement développées dans le cadre du cours de Niveau II, qui tend à attirer les meilleurs participants et aussi les plus brillants. Ces compétences sont aussi une partie essentielle du programme du Groupe spécial. Les participants à CMA Vanguard/Niveau III sont censés organiser de telles activités sous forme de débats publics et intervenir dans des incidents particuliers, par exemple en contestant une remarque désobligeante envers les femmes et en s'efforçant d'aider les autres à en comprendre l'injustice. Les participants ont, à plusieurs reprises, informé CM A quand une famille dans le quartier projetait de faire subir la mutilation sexuelle à leur fille. Madunagu signale que les responsables de CMA ont pu persuader quelques familles de ne pas faire pratiquer l'intervention.

Toutefois, CMA et les garçons ont appris que les interventions peuvent devenir compliquées et que certaines situations violentes peuvent être dangereuses pour les garçons eux-mêmes. Dans un des 
cas, un élève du N iveau II a signalé qu'une fille du quartier allait subir la mutilation sexuelle. Le personnel de CMA a envoyé une lettre à la famille de la fille et parlé à ses parents. Ce contact a apparemment empêché la mutilation, mais le père du garçon l'a chassé de la maison pendant une semaine pour « avoir causé des problèmes », et la famille de la fille l'a harcelé pendant plusieurs semaines, le qualifiant $d^{\prime}$ ' « indicateur ». Le garçon est allé demander de l'aide au conseiller de CMA et le calme a fini par se rétablir.

Chaque année en octobre, CM A organise une tribune un samedi pour les parents et tuteurs des garçons de CM A afin de leur fournir de plus amples informations sur le programme et de savoir dans quelle mesure le programme a changé leurs fils. Les parents ont suggéré que CMA commence une émission de radio et organise des débats en plein air à l'intention du public. $3 \mathrm{D}$ ans l'ensemble, la présence à la tribune des parents a été décourageante, peut-être parce que les responsables de terrain n'attirent pas suffisamment l'attention des parents sur l'événement et parce que ces derniers sont trop occupés.

Chaque année, les participants au Niveau II et au Groupe spécial sont invités à visiter des institutions et entrep rises pour être mieux informés sur la vie économique et l'histoire de la région. L'année passée, par exemple, les élèves ont visité la radiodiffusion de Cross River, le musée national, l'autorité portuaire du Nigéria et la zone de libre échange de Calabar. La participation à ces excursions est plus faible que prévue pour des raisons qui demeurent peu claires.

Enfin, le programme publie un bulletin trimestriel, « L'adolescent de sexe masculin » (TMA, The Male Adolescent), avec un financement de la Fondation MacArthur et de I'IW HC. Le but est de diffuser le message de CM A au grand public. TM A est produit par le personnel de CM A avec la contribution des élèves. Le bulletin comprend des comptes rendus et photos d'événements de CMA, des articles sur des sujets d'actualité (par exemple la violence familiale, la démocratie au Nigéria, la santé et les droits sexuels, quoi faire en cas de viol), des articles de fond sur les compétences (y compris les conseils socio-psychologiques, la facilitation et la formation), des critiques de livres et un éditorial de Madunagu (par exemple « La politique à l'ère de la haine »). Bien que le

\section{Le bulletin trimestriel de CMA suscite des discussions entre père et fils.}

contenu soit intéressant, la présentation est dense et académique, et la qualité de la mise en page et de la production est plutôt médiocre. N éanmoins, TM A touche plusieurs personnes en dehors du programme, notamment les parents. Plusieurs garçons de CMA m'ont dit que leurs parents lisent le bulletin et qu'il suscite des discussions entre père et fils.

\section{Des êtres humains transformés}

Pendant ses six premières années, CMA a conféré un diplôme à environ 2000 garçons et jeunes hommes à Calabar et Uyo. En 2002, plus de 700 garçons étaient inscrits-600 au Niveau I (30 garçons de chacun des 20 établissements d'enseignement secondaire participants), 40 au N iveau II, 15 au Niveau III et 25 au Groupe spécial. Madunagu, les donateurs d'aide à CMA et autres personnes s'intéressant au rôle des hommes dans la promotion de la santé et des droits des femmes désirent tous comprendre l'effet que le programme a sur les garçons qui y participent. De telles conclusions sont particulièrement saillantes parce que le modèle CMA est différent de la plupart des programmes d'éducation communautaires pour adolescents de sexe masculin.

\footnotetext{
3 Les dirigeants de CMA projettent désormais d'organiser trois débats publics de ce type par an-sur les droits des femmes comme droits individuels, sur l'anti-sexisme et la responsabilité des hommes ainsi que sur la démocratie et les droits des la personnes.
} 


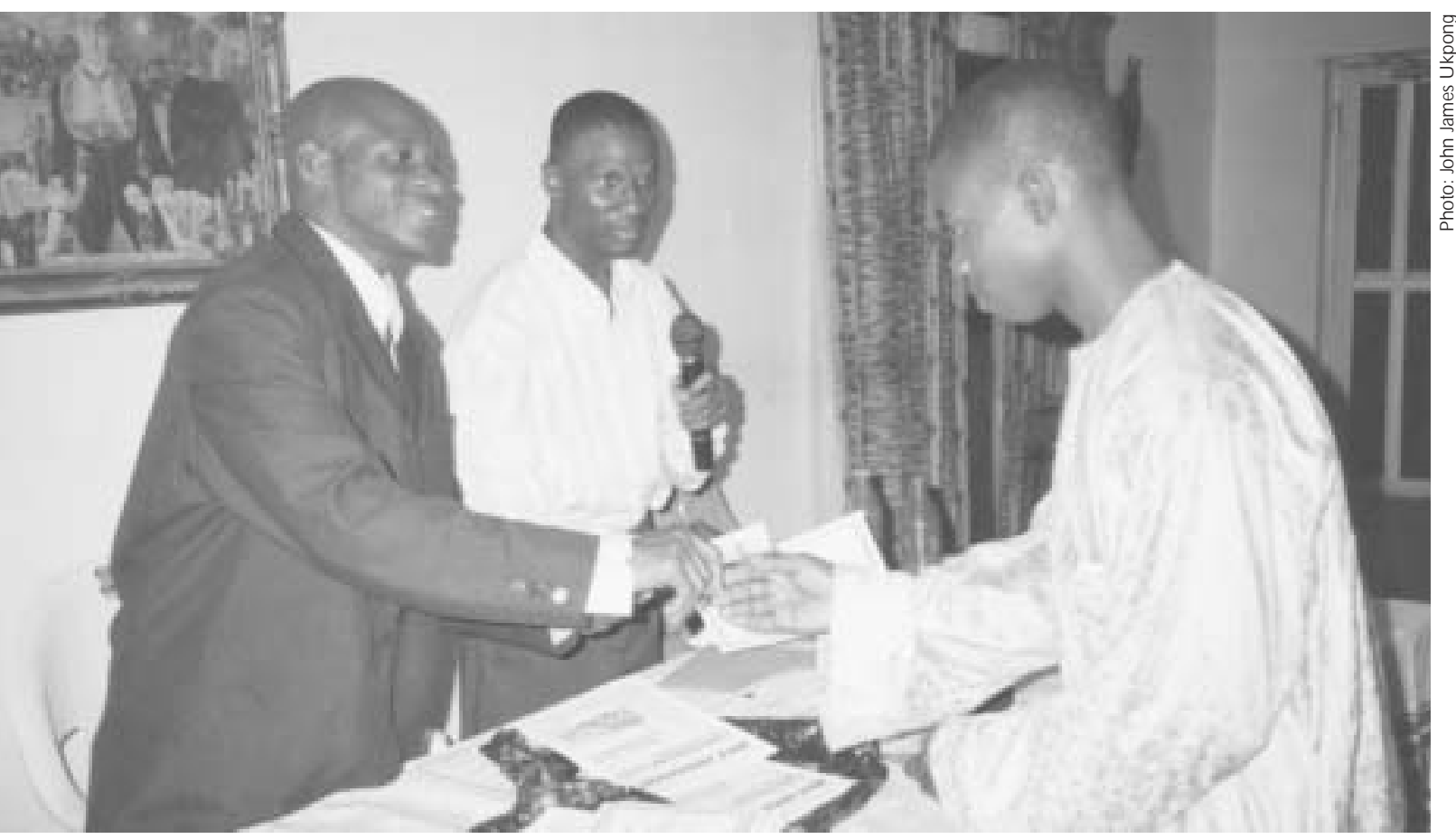

Environ 2000 garçons et jeunes hommes à Calabar et U yo ont reçu un diplôme de CMA pendant les six premières années du programme.

Les responsables de CM A n'ont pas fait d'évaluations systématiques des attitudes et pratiques des participants, mais ils essayent d'évaluer l'efficacité du programme grâce à des questionnaires concernant les connaissances et attitudes ainsi que des visites à domicile des parents (voir "Les défis », ci-dessous). Pendant ma visite, j'ai effectué des entretiens en profondeur avec dix garçons de CMA ainsi qu'avec le personnel et les membres de la communauté. Les garçons qui restent fidèles au programme disent qu'ils ont beaucoup changé. Plusieurs d'entre eux ont expliqué qu'avant leur participation à CMA, ils auraient été trop timides pour me parler. Maintenant, ils peuvent s'exprimer, présenter des arguments pour soutenir leurs opinions et intervenir quand ils ne partagent pas le point de vue d'une personne. Leur aptitude à défier les adultes ou à parler en leur présence va à l'encontre de normes sociales profondément enracinées, même pour des jeunes gens de sexe masculin. Cette aptitude à exprimer leurs opinions a contribué à faire des garçons de CMA des « vedettes » dans leurs écoles, déclare Helen Kanu, une animatrice de GPI. Par ailleurs, la connaissance inspire un profond respect au Nigéria, ce qui augmente le prestige de CM A. Les garçons sont admirés et tendent à être populaires. Ils disent qu'ils apprécient la camaraderie et les amis que CM A apporte, bien qu'ils aient tous aussi des amis en dehors de CM A.

\section{Avec leurs propres mots : les garçons de CMA réfléchissent}

Les diplômés de CMA que j'ai interrogés étaient âgés de 16 à 25 ans. D eux d'entre eux n'avaient terminé que le $\mathrm{N}$ iveau I, quatre avaient achevé le Niveau II, trois s'étaient inscrits à CM A Vanguard/N iveau III et un était dans le Groupe spécial. II n'était pas étonnant de constater que les élèves plus avancés comprenaient et exprimaient beaucoup mieux les idées de CMA que les garçons du Niveau I, plus jeunes. Dans les extraits qui suivent, les garçons de CMA discutent de l'influence du programme sur leur identité et leur vision du monde. ${ }^{4}$ 


\section{À propos de l'égalité entre les sexes}

La plupart des garçons étaient désireux d'exprimer leurs opinions sur la discrimination à l'égard des femmes en matière de politique et d'éducation ainsi qu'au foyer, et sur les raisons pour lesquelles les femmes doivent être considérées et traitées de la même manière que les hommes.

Les femmes sont politiquement marginalisées. Une seule femme est gouverneur adjointe d'un état et il y a un problème avec le gouverneur parce qu'il est sexiste. ... Le fait que le Nigéria soit patriarcal n'aide pas au développement du pays. Les parents disent aux filles de se marier, ils pensent que ça ne vaut pas la peine d'assurer leur instruction. Ils dépensent beaucoup d'argent pour faire instruire les garçons, mais ce ne sont pas tous les garçons qui réussissent. Les femmes ne sont pas censées être écoutées. Si une femme parle, on lui demande de s'asseoir. ...

Moses, 16 ans, diplômé des Niveaux I et II

La plupart des gens pensent qu'il est impossible qu'une femme soit présidente du Nigéria. Et je me dis, pourquoi pas en 2003 ? C'est possible, parce que ce ne sont que les organes reproducteurs qui font la différence entre les hommes et les femmes. Les femmes peuvent faire les mêmes choses que les hommes.

Abu, 17 ans, diplômé du N iveau II

Avant, je ne savais pas que les femmes avaient le droit d'exprimer leur opinion dans la famille. Maintenant, je le sais. Je pensais que seules les femmes devaient se lever de bonne heure le matin pour travailler et que les hommes pouvaient dormir. Maintenant, je me lève de bonne heure, avant ma sœur ; je balaie le sol etj'aide. Ce ne sont pas seulement les femmes qui doivent balayer le sol. . . - Ma mère a essayé de m'empêcher, mais je lui ai expliqué. Maintenant, elle est fière de ce que je fais.

\section{Felix, 18 ans, diplômé du N iveau II}

\footnotetext{
4 Les noms de tous les garçons et de tous les parents ont été changés.
}

Lorsqu'il s'agit de gouvernement et de politique, il faut donner aux femmes l'occasion de contribuer. Elles ont peut-être de meilleures idées que les hommes.

Kingsley, 18 ans, diplômé

du Niveau I

\section{À propos du harcèlement sex- uel et de la violence contre les femmes}

Les garçons comprennent que leur comportement passé, qui consistait à harceler et toucher les filles, était une violation des droits de celles-ci. Quelques garçons ont parlé de «protéger les filles », une approche plutôt patriarcale du sujet.

Mes amis qui ne sont pas à CMA. . . quand ils voient une fille passer, ils chuchotent des choses embarrassantes, touchent la fille et lui disent des choses désagréables. On nous a déjà dit, à certains d'entre nous qui avons été à CMA, de ne pas faire ça parce que les filles sont gênées.... J'ai arrêté de chuchoter, de me mettre sur la route et de toucher les filles n'importe comment... Je m'abstiens de faire ces choses maintenant. . . .

Kingsley, 18 ans, diplômé du N iveau I

\section{Dans le cadre d'une réflexion sur l'amour et le mariage, plusieurs garçons ont affirmé désirer une relation très différente de celle que leurs pères ont avec leurs mères. IIs ont particulièrement fait part de leurs inquiétudes concernant le mariage précoce et la polygamie.}

Voici ce à quoi le monde doit ressembler, d'après moi. Veux-tu un monde de paix ? Veux-tu un monde où règne l'équité ? Ou préfères-tu vraiment un monde où nos pères battent nos mères? Un monde où nos frères battent leurs copines et où nos sœurs sont battues par leurs copains ?. . . La culture, c'est 
quelque chose de dynamique, pas quelque chose de statique. Nous ne cherchons pas à jeter à terre notre culture. Mais il y a des aspects de notre culture qui sont négatifs. C'est contre cela que nous nous battons.

Paul, 21 ans, diplômé des

Niveaux I et II, maintenant membre du personnel de CMA

Q uelques garçons éprouvaient encore des sentiments mitigés à propos des femmes, de la violence et des rapports sexuels. Ils considéraient les femmes et les filles comme des "tentatrices », mais exprimaient aussi une certaine compréhension :

J'ai vu un homme lutter avec une fille à l'université. Je suis allé lui parler et quatre autres garçons sont venus, alors le type a fui. J'ai dit à la fille de changer sa façon de s'habiller. C'était la nuit. «II fait sombre, si vous portez ce genre de robe, d'autres hommes vont vous harceler. Le jour, vous pouvez vous habiller comme vous voulez. . . . » II y a des femmes qui vont là [dans les buissons] pour se donner à des hommes pour de l'argent, quelque part tout près. Si les femmes cessaient d'y aller, cela résoudrait beaucoup de problèmes. Mais il n'y a pas d'emploi dans ce pays, même pour les personnes instruites. C'est pour cela qu'elles font ça, peut-être. Ce n'est pas juste. Ce n'est pas bien.

Emeka, 21 ans, diplômé du Niveau I

\section{À propos des relations sexuelles précoces et de la santé sexuelle}

Tous les garçons m'ont parlé des grossesses non désirées et de la nécessité de s'abstenir d'avoir des rapports sexuels s'ils n'étaient pas prêts. Un thème commun était qu'il est possible de se lier d'amitié avec les filles sans forcément avoir de rapports sexuels, une opinion apparemment peu répandue parmi les jeunes hommes au Nigéria :

N ous discutons de ces questions à CMA, nous parlons des copines et des copains. Beaucoup de garçons ont des rapports sexuels, mais puisqu'ils ne savent rien, les filles tombent enceintes. Les garçons

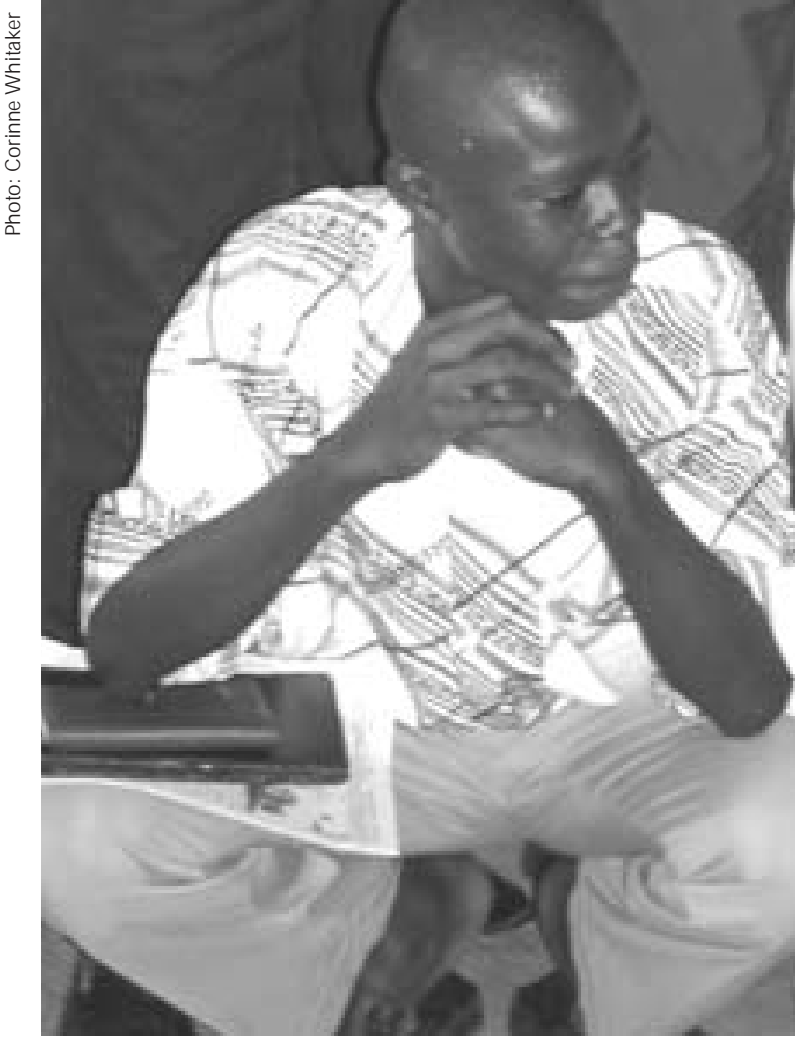

doivent réfléchir. ... Ils peuvent être amis avec des filles sans avoir de rapports sexuels. II ne faut pas avoir de rapports sexuels si on n'est pas prêt.

Emeka, 21 ans, diplômé du Niveau I

La plupart des garçons avaient des connaissances sur la santé sexuelle et reproductive, mais le niveau de leurs connaissances variait. Ce sujet met apparemment mal à l'aise certains enseignants du Niveau I. Un garçon du Niveau I a dit qu'il n'avait rien appris à CMA sur la santé sexuelle et reproductive, les préservatifs, le VIH ou autre sujet de cette nature. Les garçons du Niveau II ont déclaré qu'ils avaient discuté de la nécessité de la planification familiale et qu'ils étaient renseignés sur les contraceptifs oraux, les DIU et les préservatifs, aussi bien que sur les infections sexuellement transmissibles, y compris le VIH.

\section{À propos de l'éducation et des perspectives d'avenir}

Je ne sais pas comment je vais obtenir une licence à l'université. II y a tellement de grèves. Les enseignants n'envoient pas les résultats des examens. Je dois courir après eux pour les résultats. 
C'est ce que je fais depuis quelques jours. Je me demande à quoi ressemblera la vie. Aurai-je un emploi raisonnable ? Et si oui, voudrai-je me fixer et me marier ? Comment devrai-je m'y prendre ? La communauté me laissera-t-elle être moi-même, et prendre mes propres décisions?

Victor, 25 ans, Groupe spécial

\section{À propos du mariage et de la paternité}

La plupart des répondants avaient beaucoup réfléchi à l'amour et au mariage. Plusieurs d'entre eux ont affirmé désirer une relation très différente de celle que leurs pères ont avec leurs mères. Ils ont particulièrement fait part de leurs inquiétudes concernant le mariage précoce et la polygamie :

Par exemple, si un jour j'épouse une femme et que je décide de divorcer d'avec elle, et qu'elle a un enfant de moi, que vais-je faire ? Je veillerai à m'occuper de cet enfant et à assurer aussi une vie confortable à la mère. . . . Je veillerai à mettre fin à la relation de telle sorte que la femme ne soit pas blessée.

Mon père pensait autrement. II renverrait la femme et l'enfant et ne voudrait rien savoir. C'est en fait ce qu'il a fait avec sa première femme et ses enfants [les demi-frères et sœurs de Harry]. . . . C'est à cause de CM A que je serai différent.

\section{Harry, 20 ans, diplômé des Niveaux I et II, maintenant membre de CM A Vanguard}

Je n'aurais pas plus d'une femme à cause du problème auquel mon père est maintenant confronté. II regrette d'avoir épousé plusieurs femmes. Les enfants de mariages différents ne sont pas traités de la même façon.

Felix, 18 ans, diplômé du N iveau II

Je serai un mari différent [de mon père]. Je ne choisirai pas n'importe quelle femme. Nous devons sortir ensemble. Nous devons nous mettre d'accord sur certains points. Nous devons nous comprendre et nous entendre sur ce que seront nos vies. Je dois comprendre ma femme, je dois connaître ses sentiments et nous devons être d'accord sur presque tout. Je serai à ses côtés. Nous nous partagerons les travaux domestiques, je la soutiendrai hors du foyer, elle sera libre de faire ce qu'elle veut, je ne la retiendrai pas, je la laisserai explorer son monde à elle. Je ne suis pas la seule chose qui ait de l'importance.

Victor, 25 ans, membre du Groupe spécial

\section{Qu'en est-il du com- portement? Les filles et les adultes parlent des garçons de CMA}

À l'exception d'une école à Calabar, tous les établissements participants sont mixtes, fournissant ainsi un environnement immédiat pour tester les changements d'attitude des garçons. J'ai parlé à quelques filles de GPI, à des femmes adultes de GPI et de CMA ainsi qu'à un responsable communautaire du Sud de Calabar. Elles avaient constaté des changements remarquables dans les comportements et les attitudes des garçons de CM A à l'égard des filles et des femmes.

Je connais plusieurs garçons de CMA, y compris un de mes voisins. Avant, ils avaient plusieurs copines et disaient qu'on pouvait se servir du sexe faible. . . ils faisaient des remarques déplaisantes quand une fille passait. Maintenant, ils restent fidèles à une copine et ils ne la dénigrent pas. Ils ne harcèlent plus les filles. L'un d'entre eux, qui est étudiant à I'université, est féministe. II travaille sur les droits des femmes dans le monde entier, sur tous les aspects de ces droits, politique, économique, prise de décision. . . . Grace, 18 ans, membre de GPI, étudiante à l'université

Je connais un garçon de CMA à l'école. II ne se comporte pas comme les autres garçons. II peut parler en public et dire aux garçons comment éviter les maladies sexuellement transmissibles, le VIH et le SIDA. ... Je l'ai vu le faire. Avant CMA, il se comportait comme un ignorant. ... II ne pouvait pas parler en public, il était très timide, mais il harcelait les filles.

Veronica, 16 ans, membre de GPI, élève du secondaire 
Les adultes- parents, responsables et personnel de CMA-sont d'accord avec Grace et Veronica (voir page 23). Plusieurs parents étaient initialement sceptiques en ce qui concerne l'inscription de leurs fils à CM A en partie parce que certains jeunes gens deviennent membres de groupes considérés comme des sectes. Ce n'est qu'après avoir écouté leurs fils et vu les changements survenus dans leurs comportements que ces parents ont changé d'avis. Plusieurs parents dont les fils participent au programme depuis trois ans ont fait des remarques sur le changement :

Vous savez que nous vivons dans une zone dominée par des «bandes » de garçons du quartier et, évidemment, la question des sectes dans les écoles sévit actuellement. J'ai donc pensé qu'il essayait de camoufler la vérité. . . . [Puis] la première chose que j'ai observée c'est qu'il a commencé à faire la cuisine à la maison ... parce que les garçons ne sont pas censés faire ce genre de choses. Je lui ai demandé où il avait appris cela et il m'a répondu que c'était au programme CMA. Je lui ai alors demandé ce que CMA signifiait et il me l'a dit. Deuxièmement, j'ai constaté qu'il devenait plus responsable, c'est-à-dire qu'il n'attendait plus qu'on le pousse à faire quelque chose. Là encore je lui ai demandé pourquoi ce changement subi de comportement, et il a dit que c'était grâce à CMA. J'ai alors dit Dieu merci, mais j'étais toujours sceptique en ce qui concerne ce programme. Depuis, je me suis rendu compte que le programme CMA est conçu pour corriger les maux qui règnent dans la société.

M. Uwem Esu

Avant, il se passait à peine une semaine sans que les voisins viennent se plaindre de son mauvais comportement [de mon fils], par exemple ses bagarres avec le fils du voisin. Même l'école m'envoyait beaucoup de mauvais rapports sur lui. Mon fils m'a dit que CM A était un programme éducatif sur les droits des femmes et je lui ai tout simplement dit de faire attention de ne pas devenir membre d'une secte, puisque c'était un garçon très têtu. Maintenant, il est complètement différent, il est toujours à la maison et il prend ses études plus au sérieux que jamais auparavant. II informe maintenant d'autres personnes sur la nécessité de mettre fin à la violence contre les femmes.
Dans cette maison, nous avons un tableau pour les travaux domestiques. Auparavant, chaque fois que c'était le tour [de mon fils] de faire des tâches domestiques, il se montrait très têtu. Maintenant, non seulement il participe volontairement aux travaux domestiques, mais c'est lui qui dresse la liste et veille à ce que les autres s'y conforment. Je ne suis pas toujours à la maison parce que je suis commerçante, mais j'ai aussi remarqué que chaque fois qu'il rentre du programme CMA, il partage l'information avec d'autres personnes à la maison, surtout sa sœur.

$\mathrm{M}$ me Comfort A to e

C'était un garçon très timide. J'ai remarqué avec un vif intérêt qu'il a maintenant son franc-parler. II a pu inculquer un élément d'amour-propre à ses sœurs surtout, en utilisant ces bulletins, mais encore plus par sa façon de parler.

La première fois que je suis tombé sur le bulletin d'information de CMA, j'étais effrayé à cause de la question de la « conscience critique ». Je pensais que [ces] gens étaient bien décidés à produire des voyous et des enfants qui viendraient se battre contre leurs parents à la maison, tout cela au nom de l'éducation. J'ai dû dire à mon fils de faire attention à ses fréquentations, sinon il se retrouverait en prison. Maintenant, je pense que [CM A est en train de] faire du très bon travail dans la vie de ces adolescents en les façonnant pour en faire des adultes responsables. Je le dis à cause de la transformation de la vie de mon fils et parce qu'il peut utiliser cette information pour influencer la vie de ses sœurs. Je peux à présent dire que CM A est résolu à transformer les personnes et non à les détruire.

M. Charles Ibiam 
Les responsables communautaires observent les garçons de CMA hors de chez eux, et ils les décrivent ainsi:

II y a environ 12 garçons de CMA dans ma communauté et ils sont actifs, très actifs. Ils lisent beaucoup. Ils ne lisaient pas avant, mais maintenant ils lisent beaucoup. Ils ont une influence sur des centaines de garçons. Avant, quand les filles allaient chercher de l'eau, des garçons les attendaient pour leur toucher les seins et le derrière, pour les mettre mal à l'aise. ... Les garçons de CM A ont organisé une réunion sur le thème « Aimer une enfant ». Ils ont invité un échantillon de garçons, d'hommes et de filles. En fin de compte, nous avions environ 500 personnes dans la salle des fêtes. D urant la discussion, il était question de considérer les filles comme des êtres humains . . . en pensant à sa sœur, à sa mère. Cette discussion a rapproché les garçons des filles.

Sandra, responsable communautaire

Je vois une très grande différence. . . . Si les jeunes garçons sont conscientisés et éduqués, cela prépare le terrain pour le travail de [GPI] et pour la société en général ... quand ils communiquent avec les membres du sexe opposé, ils incorporent les femmes dans la prise de décision. . . . Cela facilite les relations. . . . Les filles de GPI communiquent avec les garçons de CMA. Les garçons de CMA comprennent qu'ils peuvent être amis avec les filles, qu'il ne s'agit pas seulement de rapports sexuels. Les filles peuvent discuter avec les garçons de CMA et s'exprimer.

Helen, animatrice de GPI

Des directeurs d'école viennent ici pour s'informer sur le programme et demander qu'un plus grand nombre de leurs élèves y participent. La nouvelle directrice de la West African Peoples' Institution a vu la manière dont les garçons de CMA intervenaient dans les questions concernant les filles et elle était impressionnée. Elle m'a dit qu'elle veut que plus de 30 de ses élèves soient inscrits au programme.

Winifred, membre du personnel de CMA
CMA a cet impact parce qu'il vise les garçons à l'âge où ils peuvent le plus facilement changer.

Igwe Dermot, coordonnateur des responsables de terrain de CMA

Il semble que l'influence des garçons de CMA sur leurs pairs change progressivement les normes prévalentes de comportement social à l'école. Un enseignant à Calabar a signalé qu'un petit groupe de durs harcelaient constamment les filles et d'autres garçons dans son école. L'un d'entre eux, un leader né, s'est inscrit à CMA. II a depuis lors cessé de harceler d'autres élèves. Ses anciens compagnons, qui ne sont pas inscrits à CMA, ne semblent pas comprendre ses nouvelles idées, mais ils

Quand je lui ai demandé comment le fait de travailler avec seulement 30 garçons par an pouvait changer tant de choses dans un grand établissement, un enseignant a répondu que les garçons de CMA sont les plus intelligents et que plusieurs d'entre eux étant des leaders, ils donnent le ton.

voient que quelques autres garçons et lui ont changé leur comportement et prennent leurs études plus au sérieux. La pression du groupe les a progressivement encouragés à cesser de harceler les autres élèves, même s'ils ne comprennent pas nécessairement pourquoi.

Les enseignants de Christian Commercial Secondary School, un établissement secondaire mixte d'Uyo, ont signalé une transformation spectaculaire dans le comportement des garçons ainsi que dans les relations entre les garçons et les filles à la suite de CMA. Selon un enseignant, avant le programme CMA, les garçons harcelaient tellement les filles qu'elles refusaient de s'asseoir à la bibliothèque scolaire. Maintenant, il voit les garçons et les filles s'asseoir ensemble, silencieusement, 
et il secoue la tête avec stupéfaction. Q uand je lui ai demandé comment le fait de travailler avec seulement 30 garçons par an pouvait changer tant de choses dans un grand établissement, il a répondu que les garçons de CMA sont les plus intelligents et que plusieurs d'entre eux étant des leaders, ils donnent le ton.

Un autre enseignant a raconté que la sortie d'une école de filles tout près a lieu presque au même moment et que par le passé, il y avait beaucoup d'incidents de harcèlement. Aujourd'hui, le harcèlement a remarquablement diminué. Les enseignants m'ont aussi dit que les filles de leur établissement ont entendu parler de CMA et qu'elles ont demandé que des groupes de G PI soient établis à leur intention. GPI travaille maintenant dans plusieurs écoles d'U yo.

L'impact des enseignants de CMA comme modèles de comportement au sein des écoles a aussi été mentionné dans les entretiens que j'ai effectués. Le harcèlement des élèves du sexe féminin par les enseignants hommes est courant

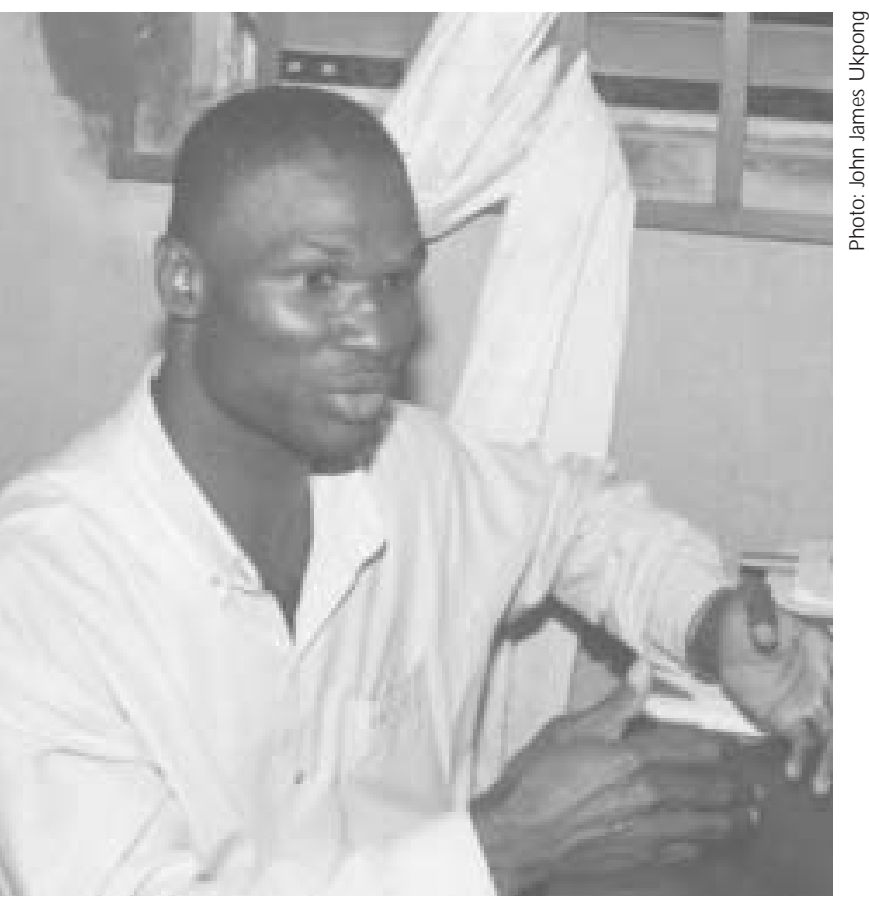

Les responsables de terrain, comme Alphonsus Ekah, déclarent que le fait de travailler à CMA a eu un énorme effet sur leurs propres croyances et comportement. dans les écoles nigérianes. Les garçons (et les filles) sont intrigués par les enseignants de CMA, qui prêchent par l'exemple. Beaucoup de garçons vont leur demander d'être leurs guides et conseillers.

\section{Les enseignants comme apprenants}

Les responsables de terrain de CMA, qui dirigent les groupes de discussion, affirment avoir beaucoup appris du programme et l'enseignent avec enthousiasme. Certains d'entre eux déclarent que le programme a énormément changé leur propre comportement.

O ui, j'ai été merveilleusement transformé. ... . J'étais l'une de ces personnes qui pensaient qu'une femme n'a pas le droit de dépenser mon argent sans rien donner en retour. Pas que je la violerais, ... mais je ferais tellement pression sur elle que ... puis progressivement, vous vous rendez compte qu'après tout, la femme n'éprouve aucun plaisir, et que vous avez fait du tort à quelqu'un . . .

Charles, enseignant, ancien coordonnateur des responsables de terrain de CMA, animateur

Ce programme ira vraiment loin. Je suis très passionnée par ce que nous faisons. ... C'est pour moi un avantage d'être une femme et de diriger les discussions. Je leur raconte des histoires sur ma famille et ma vie. Ils aiment ces histoires. Ils me posent des questions. Ils communiquent très librement avec moi. Si j'avais eu ce genre d'information à 15 ans [elle s'est mariée à 15 ans et a cinq enfants], ma vie aurait été différente.

Mercy, membre du personnel de CMA, animatrice pour le N iveau II et le Groupe spécial

Avant, je pensais que les seules relations qu'on pouvait avoir avec une fille étaient des relations sexuelles et qu'un garçon ne pouvait pas communiquer avec une fille sans qu'il y ait l'aspect sexuel. . . . J'ai beaucoup changé.

Alphonsus, 27 ans, membre du personnel de CMA (est devenu membre du personnel en 1996, à 21 ans) 


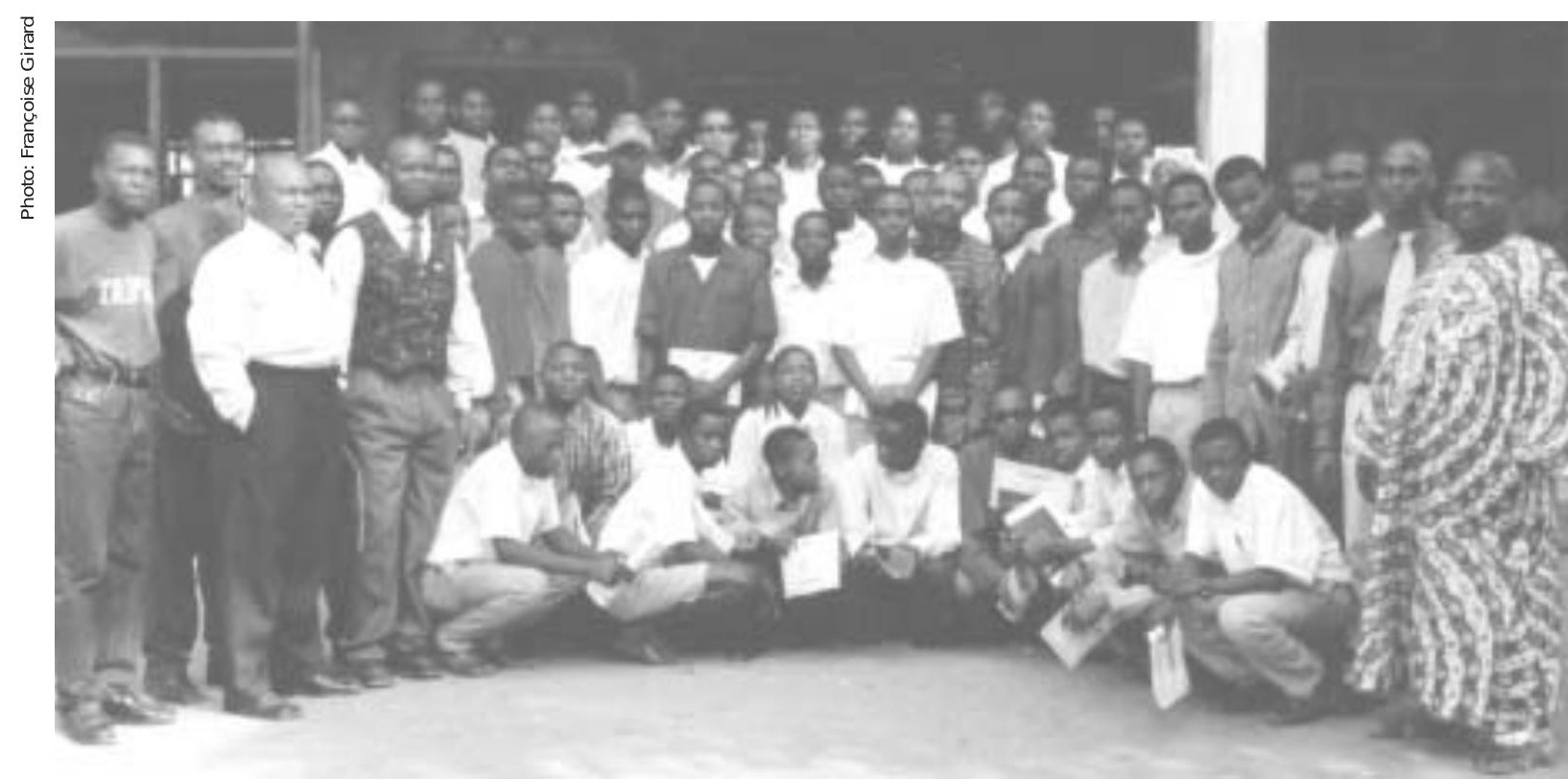

Les 2000 diplômés de CMA étant en général exceptionnellement compétents et influents, l'effet du programme est considérablement multiplié.

\section{CMA comme agent de changement social}

Quand Madunagu a fondé CMA, son objectif était de créer un complément masculin de Girls' Power Initiative. Avec le temps, sa vision de l'organisation a évolué. II considère maintenant le travail de CMA-encourager la conscience antisexiste et critique-comme un important aspect de la lutte pour la démocratisation du Nigéria, et ceci pour trois raisons : premièrement, CMA affecte un nombre de plus en plus grand de garçons et l'offre est inférieure à la demande. Deuxièmement, les 2000 diplômés de CMA étant en général exceptionnellement compétents et influents, l'effet du programme est considérablement multiplié. Troisièmement, le personnel de CMA a approfondi sa compréhension de la manière dont le travail anti-sexiste touche aux problèmes sociaux en général.

Madunagu est conscient du fait qu'informer les garçons et les jeunes hommes sur le sexisme et encourager la pensée critique sont des activités qui peuvent sembler menaçantes aux yeux de certaines personnes. II déclare à cet effet :
«Évidemment, l'oppression et le pouvoir sont les principaux sujets dont nous parIons à CMA. Dans ce pays, beaucoup de gens croient qu'on est soit opprimé soit oppresseur. ... O u vous marchez sur les pieds de cette personne, ou cette personne vous marche sur les pieds. ... M archer côte à côte est une chose que nous ne savons pas faire. »

CMA s'est délibérément tenu à l'écart de la politique organisée, en dépit-ou peut-être précisément à cause-du passé de Madunagu. Pour une organisation qui travaille avec les garçons, devenir ouvertement partisan pourrait être perçu comme une menace politique, et entraîner la fermeture de CM A par les autorités de l'État. Des individus qui pourraient avoir des rapports avec les forces de sécurité viennent tout de même régulièrement s'asseoir à la bibliothèque de CIINSTRID pour observer silencieusement les activités.

Quand j'ai demandé à Madunagu ce que la plupart des gens pensent de son travail, il a simplement ri : " Ils pensent que ce que je fais est très bizarre, ce qui a confirmé mon excentricité dans leur esprit. Mais en même temps, je suis très respecté. » 


\section{Les défis}

La croissance rapide de CMA dans un domaine en grande partie inexploré n'est pas allée sans difficultés. Les entretiens avec Madunagu, les responsables de terrain et les garçons de CMA ont révélé une série de questions épineuses-pédagogiques, institutionnelles et politiques.

\section{Perfectionner les responsables de terrain}

Souvent, les responsables de terrain évoluent en même temps que leurs élèves et beaucoup d'entre eux « sont encore en train d'assimiler ces connaissances ». Certains ont tendance à retourner de temps en temps à l'approche didactique traditionnelle, au lieu du dialogue ouvert.

\section{A border les sujets difficilles}

Le personnel de CMA et les responsables de terrain signalent que certains sujets sont particulièrement difficiles à aborder pendant les discussions ou la formation des enseignants. Madunagu pense qu'ils doivent préparer une meilleure documentation sur ces questions. Ces sujets difficiles sont abordés ci-dessous.

\section{La religion et le rôle des femmes}

Le personnel de CMA révèle que les garçons de familles pieuses ont souvent du mal à concilier ce qui leur a été dit dans leur pratique religieuse avec l'idée d'égalité entre les sexes. 5 En effet, dans les questionnaires administrés aux garçons au début du programme, la majorité des répondants ont affirmé que l'homme est le chef de famille parce que c'est ce que dit la Bible. De tels enseignements religieux appuient les normes sociales prévalentes et, par conséquent, semblent indéniablement « vrais » à beaucoup de garçons. Un garçon a prétendu que les hommes ont de multiples partenaires parce que "la Bible dit qu'un homme peut épouser 500 femmes».

Les opinions de beaucoup de garçons sur la sexualité, la masturbation, l'abstinence et l'avortement sont profondément influencées par les enseignements religieux, mais CMA insiste sur le pluralisme comme principe directeur. Le contenu du programme est entièrement laïque et les arguments fondés sur les textes religieux ne sont pas admissibles dans les discussions. Certains garçons ont quitté CMA parce qu'ils ne pouvaient pas concilier leurs croyances religieuses avec les enseignements de CMA et, selon les rapports des dossiers des services de conseils, ceux qui continuent à suivre le programme semblent être aux prises avec cette discordance. Igwe Dermot a expliqué que certains garçons auraient voulu parler de ce qu'ils apprennent à CMA avec leurs parents, mais ils pensent qu'ils ne peuvent pas soulever certaines questions, notamment la sexualité, à cause des croyances religieuses de leurs parents.

\section{La masculinité}

Les garçons que j'ai interrogés n'avaient pas explicitement réfléchi à la définition sociale de la masculinité et ses écueils d'un point de vue personnel ou sociétal. Ils ne pouvaient pas dire grand chose sur les pressions qu'ils subissent en tant que garçons, ce qui leur déplaît dans le fait d'être des garçons, ou dans quelle mesure d'autres garçons ou hommes pourraient les faire souffrir. Paul Awah a indiqué que dans certaines discussions qu'il a menées, les garçons ont fait des remarques concernant certains des effets négatifs que les normes de masculinité ont sur eux. Ils ont cité, par exemple, le fait que ce sont les hommes qui doivent aller à la guerre et qui sont victimes d'assassinats politiques. Mais il reconnaît que leur analyse n'est pas encore bien développée parce que « les garçons ne sont pas prêts à dire que le fait d'être des garçons leur cause des problèmes ».

\footnotetext{
${ }^{5}$ Dans l'Est du Nigéria, le christianisme et les religions traditionnelles prédominent.
} 


\section{Blâmer la victime de la violence sexuelle}

Les membres du personnel m'ont dit que beaucoup de garçons continuent à blâmer les victimes du viol ou à penser que dans la plupart des circonstances, les filles et les femmes sont disponibles et qu'on " n'a qu'à se donner la peine de les prendre ». Par conséquent, ils ont du mal à comprendre et condamner le viol conjugal ou même l'inceste. La question de savoir si un homme peut être violé par une femme demeure troublante pour certains garçons. La supposition sous-jacente est que les femmes sont, d'une façon ou d'une autre, responsable du désir sexuel non contenu des hommes et qu'elles « forcent les hommes à avoir des rapports sexuels » .

\section{La santé sexuelle}

Les sujets tels que les infections sexuellement transmissibles, les expériences sexuelles, la physiologie reproductive et la négociation de la contraception sont encore des sujets tabous chez certains responsables de terrain qui hésitent à discuter de la sexualité et de la santé sexuelle en profondeur. Cette hésitation semble particulièrement concerner les enseignants du Niveau I. À la demande de CMA, GPI a dispensé aux responsables de terrain et au personnel une formation sur ces sujets, mais une formation supplémentaire est apparemment nécessaire pour assurer la qualité et la cohérence du contenu.

\section{Les con séquences sociales de la non-conformité}

Se prononcer contre des comportements aussi communément admis que la mutilation sexuelle ou la violence familiale a impliqué certains garçons et membres du personnel de CMA dans des situations compliquées et contradictoires. Les dirigeants de CMA cherchent encore des moyens d'améliorer le programme afin de préparer les garçons pour ce défi, par exemple en les aidant à développer des styles d'intervention plus efficaces. Ils espèrent aussi renforcer le service de conseils socio-psychologiques pour mieux soutenir les garçons qui subissent les répercussions de leur franc-parler.

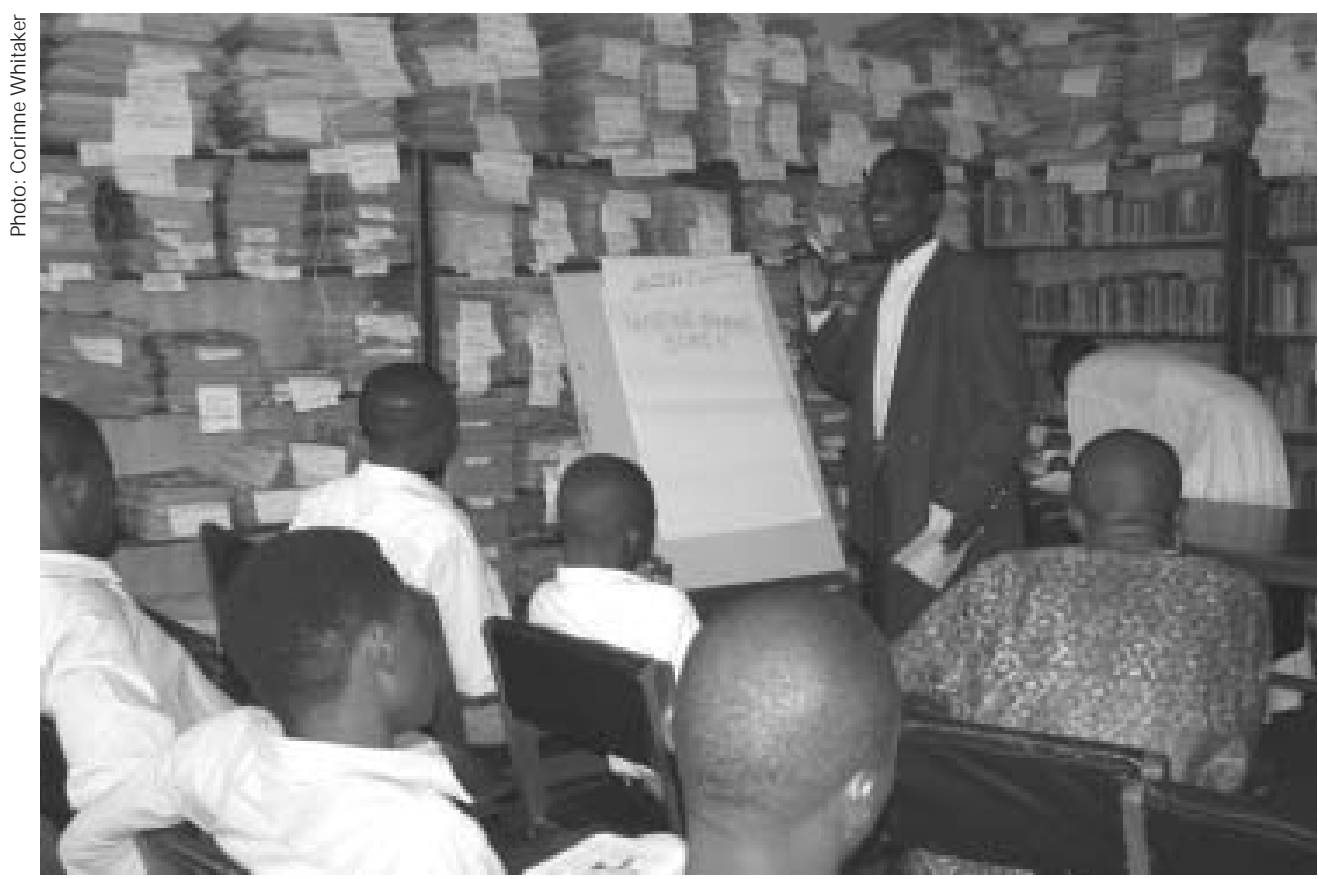

Beaucoup de garçons continuent à blâmer la victime du viol et ont du mal à comprendre et condamner le viol conjugal. 


\section{Les défis institutionnels}

Beaucoup de problèmes institutionnels auxquels sont confrontés les dirigeants de CMA sont communs aux contextes où prévalent la pauvreté et l'instabilité politique, tandis que d'autres défis se rapportent directement à la philosophie et à la méthode de CMA.

\section{A bandons}

Les taux de continuation des participants d'un niveau à l'autre sont élevés compte tenu de la durée de l'engagement requis pour participer au programme. Dans certains établissements cependant, jusqu'à 25 pour cent des garçons du Niveau I abandonnent le programme, et l'absentéisme est aussi courant. Le problème que pose à certains garçons le contenu de la discussion est manifestement un facteur. Certains garçons sont absents ou abandonnent parce que leurs parents n'ont pas payé les frais de scolarité. Le personnel de CMA signale aussi que certains garçons ont du mal à se concentrer pendant les discussions parce qu'ils ont faim après l'école. Des paquets de biscuits sont maintenant distribués dans certaines écoles. Certains garçons du Niveau II se plaignent que l'allocation de transport donnée par CMA pour se rendre aux discussions mensuelles est insuffisante.

\section{Opinions des parents}

Bien que la plupart des parents soient apparemment satisfaits de la participation de leurs fils à CM A, certains se méfient du programme, surtout au début (voir plus haut la section intitulée «Q Q 'en est-il du comportement ? ») Les responsables de CMA sont en train d'élaborer des mécanismes pour dissiper le scepticisme des parents. Madunagu a invité un père qui pensait que CMA « corromprait » son fils à venir lui parler au CIINSTRID. Maintenant, le père est heureux que son fils aille à la bibliothèque du CIIN STRID au lieu de "vagabonder». Le personnel se rend aussi compte que les publications de CMA comme The Male Adolescent rassurent la plupart des parents qu'il ne s'agit pas d'une des sectes ou sociétés secrètes qui fleurissent dans les établissements secondaires au Nigéria. Les dirigeants de CMA sont convaincus que la localisation du programme dans les écoles donne de la crédibilité au programme et diminue les inquiétudes des parents, mais que les écoles peuvent se trouver prises entre deux feux. Les enseignants organisent par conséquent des séances d'orientation dans les écoles où les parents expriment au directeur leur crainte que CMA apprendra à leurs fils à avoir des rapports sexuels.

\section{Gestion du programme}

Dès le début, les garçons de CMA ont joué un rôle essentiel dans la structure et même l'organisation du programme. Les diplômés de CMA ont commencé très tôt à travailler à CMA à temps partiel et ils ont aidé à l'élaboration du programme d'enseignement. Placer la participation des adolescents au centre du travail de CM A a manifestement contribué à l'attrait et l'efficacité du programme. Toutefois, Madunagu a au départ donné à ces adolescents des responsabilités et un pouvoir qu'ils n'étaient pas préparés à assumer, ce qui a entraîné des conflits internes parmi les membres du personnel et un certain nombre de vols.

Madunagu se rend compte que la sélection du personnel à CMA au CIINSTRID a été relativement peu méthodique. Beaucoup de membres du personnel ont été engagés simplement parce qu'ils avaient besoin d'un soutien financier. Certaines personnes engagées pour cette raison sont devenues des membres fiables du personnel qui continuent à travailler avec I'organisation. Toutefois, des conflits existent entre les membres du personnel dévoués à CM $\mathrm{A}$ et ceux qui sont là principalement parce qu'ils ont besoin d'argent.

Le personnel permanent de CMA et $M$ adunagu lui-même qualifient la gestion de CMA de «mouvementée ». Cette situation n'est peut-être pas inattendue dans une organisation qui encourage la pensée critique, les discussions ouvertes et les relations égalitaires. N éanmoins, cette agitation est en partie due au style de 
direction de Madunagu : il est pour l'autocritique et est exigeant sur le plan de l'engagement, peut-être déraisonnablement. Bien qu'il soit décidé à ce que les garçons maintiennent un rôle principal dans le programme, il compte aussi mettre sur pied une équipe professionnelle plus stable pour la gestion de base.

\section{Salaires et rotation $\mathrm{du}$ personnel}

Les Nigérians sont confrontés à une pauvreté écrasante et le niveau de vie de la classe moyenne a radicalement baissé. Beaucoup d'employés de CMA soutiennent d'autres membres de leur famille et les frais de scolarité sont un problème constant. Les familles des jeunes diplômés de CMA faisant partie du personnel, en particulier, font pression sur eux pour qu'ils contribuent de plus en plus au budget de la famille. Puisque le CIIN STRID est une organisation non gouvernementale qui reçoit de l'argent des " oyibo » (des blancs) et parce que l'accès au programme est gratuit, ses ressources sont perçues comme étant inépuisables.

En tant que penseur de gauche et activiste maintenant très lié aux donateurs étrangers et conscient de leurs attentes concernant CMA, Madunagu fait face à des dilemmes relevant de la philosophie et de la gestion. Cela le chagrine de raconter les histoires de membres du personnel qui s'exclament, quand ils lui demandent des augmentations de salaire et des prêts personnels : «Mais vous dites que vous êtes socialiste! »

La rémunération des responsables de terrain semble être un problème continuel. Malgré des augmentations régulières, ils réclament constamment plus. Madunagu déteste sa position d'employeur et les discussions d'argent. II déclare que les salaires actuels des responsables de terrain sont le double de ce qu'il avait prévu Iorsqu'il a établi le plus récent budget. II s'inquiète de ce que les animatrices de GPI gagnent deux ou trois fois moins que ceux de CMA, alors qu'elles travaillent probablement trois fois plus, ce qui est un énorme paradoxe pour deux organisa- tions qui se consacrent à l'égalité entre les sexes.

Plusieurs responsables de terrain du Niveau I ont du mal à combiner leur travail régulier d'enseignant avec le travail de CMA, qui exige formation et préparation. Certains ne se présentent pas pour les séances hebdomadaires avec les garçons. Le renouvellement des responsables de terrain dans les écoles est modéré : un responsable de terrain sur dix part chaque année, en moyenne.

\section{Expansion des activités}

L'expansion des activités demeurera un défi, étant donné l'important investissement requis pour former et superviser les responsables de terrain et le personnel.

\section{Madunagu s'inquiète de ce que les animatrices de Girls' Power Initiative gagnent deux ou trois fois moins que ceux de CMA-alors qu'elles travaillent probablement trois fois plus-ce quil est un énorme para- doxe pour deux organisations qui se consacrent à l'égal ité entre les sexes.}

De plus, CMA ne travaillant délibérément qu'avec les garçons qui font figure de leaders, l'élargissement du programme ne peut pas provenir de l'inscription d'un nombre croissant d'élèves dans chaque école ; le programme doit plutôt toucher un plus grand nombre d'écoles et de villes. Madunagu pense aussi que le programme doit renforcer la formation des responsables de terrain et améliorer le matériel pédagogique avant de s'étendre davantage. Finalement, le style de gestion que Madunagu préfère ne convient pas très bien à l'élargissement du programme ; le personnel consacre en permanence une quantité importante d'énergie à la réorganisation interne, aux changements dans les structures des comités ainsi qu'aux réunions du personnel pour discuter des problèmes. 


\section{É valuation}

Comme mentionée plus haut, le programme a utilisé deux principaux outils pour évaluer l'impact de CM A sur les garçons : les questionnaires et les visites à domicile. Les questionnaires de base permettent d'évaluer les connaissances et opinions des garçons au début des N iveaux I et II. Les questionnaires de milieu de trimestre et de fin d'année sont utilisés pour évaluer les changements dans les connaissances et les opinions déclarées. II se peut que certains élèves fournissent des réponses non sexistes aux questionnaires sans avoir vraiment changé leurs croyances et comportements. À ce jour, les questionnaires n'ont pas été systématiquement analysés. Les réponses sont simplement recopiées dans un cahier par un membre du personnel. J'en ai lu plusieurs et la teneur des réponses semble indiquer que les connaissances et opinions déclarées des garçons sont effectivement devenues moins sexistes pendant la durée du programme. Lorsque j'ai visité le programme pendant I'année scolaire 2001-02, les membres du personnel ne savaient pas très bien s'ils allaient administrer les ques- tionnaires de fin d'année et envisageaient plutôt de faire des visites à domicile.

Les membres du personnel de CMA qui sont diplômés du programme font des visites à domicile et rédigent un rapport. Les rapports ont cependant une valeur limitée. II n'y a souvent personne à la maison qui connaisse assez le garçon pour pouvoir répondre aux questions le concernant. Ainsi, le personnel de CMA rentre souvent bredouille. De toute façon, les questions posées par le personnel de CMA ne semblent pas être suffisamment précises pour produire des réponses utiles. Dans la mesure où des remarques ont été relevées, elles tendaient à indiquer que le comportement du garçon s'était amélioré. Le personnel reconnaît qu'il devra former les enquêteurs et établir un système de rendezvous précis avec les parents s'il veut continuer à utiliser cette méthode d'évaluation.

Les responsables de terrain sont également évalués de façon informelle aussi bien par le personnel, qui observe quelquefois les séances, que par les élèves dont les commentaires sont nécessaires pour déterminer l'efficacité des responsables de terrain comme animateurs de discussion.

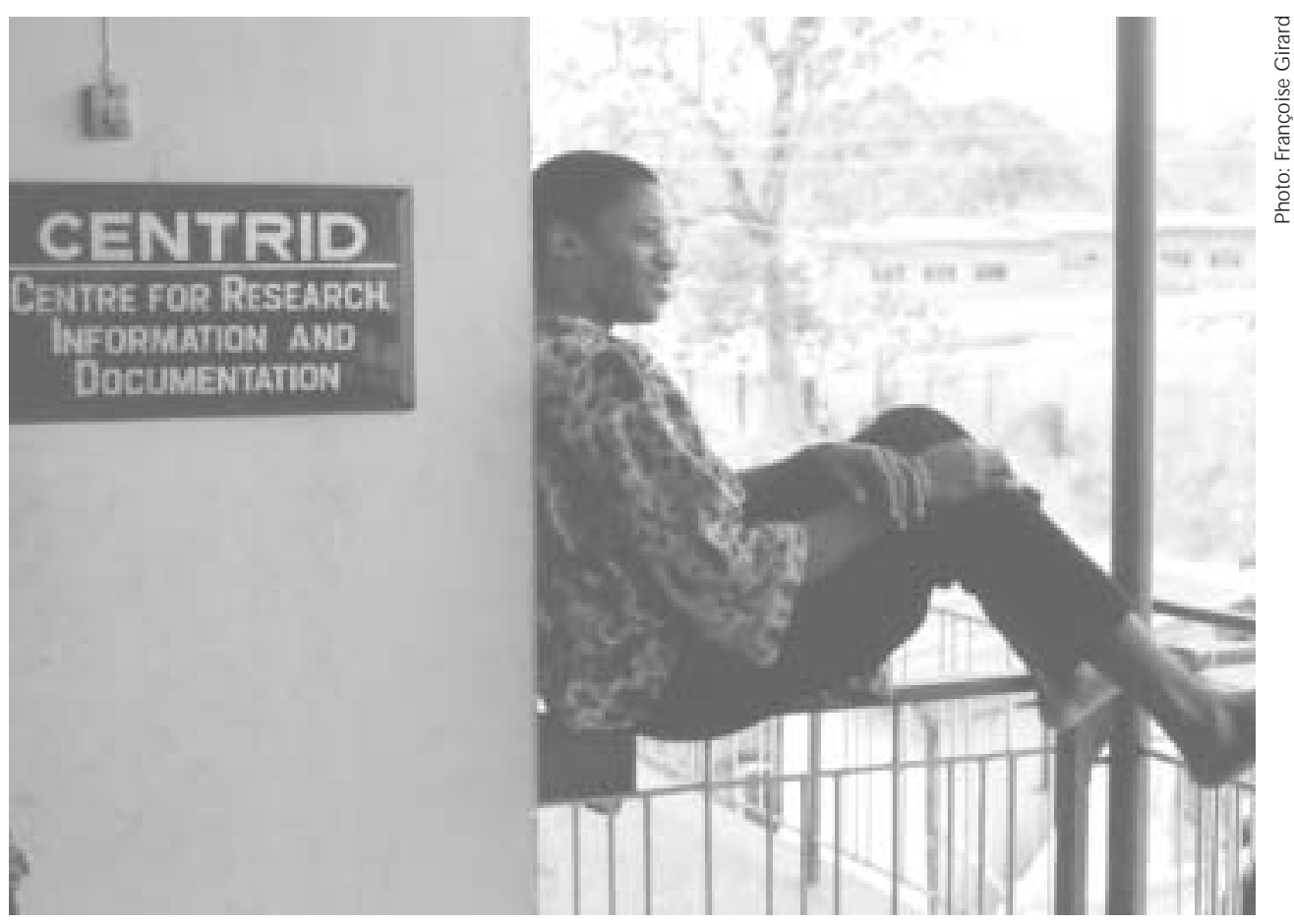




\section{Conclusions et Ieçons tirées}

Travailler avec les garçons est maintenant considéré comme une nécessité pour les personnes qui espèrent réaliser l'égalité entre les sexes et garantir la santé et les droits sexuels et reproductifs. Le programme CM A montre qu'il est possible de travailler avec les garçons sur les questions de sexisme et d'injustice sexiste, même dans un contexte d'inégalité marquée. II faudra du temps pour consolider les acquis du programme et aborder ses points faibles. Toutefois, dans un coin du sud-est du Nigéria, la « conscientisation » des adolescents de sexe masculin est manifestement plus qu'une idée, et l'expérience de CMA fournit déjà de très importantes leçons à tous ceux qui conçoivent des programmes destinés à toucher les adolescents de sexe masculin et les jeunes hommes:

- Les garçons ont soif de connaissances. Ils veulent apprendre à s'exprimer, à penser de façon critique et à comprendre le monde dans lequel ils vivent. Une formation solide et continue, en même temps que des ouvrages de référence appropriés, doivent être fournis aux animateurs pour encourager ce processus.

- Beaucoup de garçons âgés de 14 ans et plus peuvent conceptualiser des idées et finir par comprendre l'oppression des femmes par analogie, mais le processus d'apprentissage est difficile parce qu'ils n'ont pas personnellement vécu l'oppression sexiste.

- Tous les garçons ne peuvent pas bien saisir le concept d'égalité entre les sexes. Néanmoins, ils adapteront leur comportement aux normes sociales établies par leurs pairs.

- Pour les programmes cherchant à toucher les garçons particulièrement intelligents et motivés, les opportunités d'enrichissement intellectuel peuvent au moins présenter autant d'attrait que d'autres activités auxiliaires. Evidemment, les séances doivent toujours être interactives et amusantes, et les animateurs doivent utiliser diverses techniques pour communiquer le contenu.

- Les programmes doivent prendre garde à ne pas promouvoir une autre forme de patriarcat selon laquelle les garçons « protègent » les filles et les femmes.

- Des méthodes d'évaluation efficaces doivent être élaborées pour évaluer les changements dans les normes sociales et la dynamique du pouvoir au niveau de la communauté.

- Aider les garçons à penser de façon critique et étendre leurs connaissances sur le pouvoir et le sexisme peuvent être des moyens plus efficaces de produire un changement de comportement-y compris les comportements qui ont des conséquences sur la santé-que se focaliser sur la santé. Les concepteurs de programmes voudront peut-être considérer un tel changement de paradigme. 


\section{À propos des auteurs}

Gary Barker est le directeur de I'Instituto PRO MUNDO, une organisation brésilienne qui travaille avec les hommes et les garçons.

Françoise Girard est une consultante indépendante qui occupait auparavant le poste d'administratrice principale de programmes pour les politiques internationales à I'International Women's Health Coalition.

\section{Personnel de production}

\section{Directrice de la rédaction :}

Debbie Rogow

\section{Assistante de recherche \\ et de rédaction : Michelle Skaer}

Graphiste : Mike Vosika

Traducteur : Jeannette Ndong

\section{Groupe consultatif}

$\begin{array}{ll}\text { Delia Barcelona } & \text { Laura Laski } \\ \text { Gary Barker } & \text { Ann Leonard } \\ \text { Carmen Barroso } & \text { Ann McCauley } \\ \text { Judith Bruce } & \text { Liz McGrory } \\ \text { Batya Elul } & \text { Manisha M ehta } \\ \text { Susana Galdos } & \text { Suellen Miller } \\ \text { Kirrin Gill } & \text { Saumya Ramarao } \\ \text { Françoise Girard } & \text { Julie Reich } \\ \text { Nicole Haberland } & \text { Ann Starrs } \\ \text { Katherine Kurz } & \text { Cynthia Steele }\end{array}$

CIIN STRID/CM A

90B Goldie Street

P.O. Box 915

Calabar Nigéria

Téléphone : 087-234704

Fax : 087-238615

courriel: ciinstrid@hyperia.com

N ous serions heureux de recevoir vos commentaires sur Q uality/Calidad/Q ualité. Si vous souhaitez être inclus dans notre fichier d'adresses, veuillez nous envoyer un courrier électronique à l'adresse suivante : qcq@popcouncil.org. La plupart des éditions précédentes sont disponibles en ligne à l'adresse suivante : <www.popcouncil.org/publications/qcq/ default.htm>. Les ouvrages suivants sont également disponibles ; vous pouvez commander une ou plusieurs copies par courrier électronique :

Celebrating Mother and Child on the Fortieth Day: The Sfax Tunisia Postpartum Program (en anglais seulement), № 1, 1989.

Man/Hombre/Homme: Meeting M ale Reproductive Health Care Needs in Latin America (en anglais et espagnol), no 2, 1990.

The Bangladesh Women's Health Coalition (en anglais seulement), № 3, 1991.

Gente Joven/Young People: A Dialogue on Sexuality with Adolescents in Mexico (en anglais et espagnol), no 5, 1993.

The Coletivo: A Feminist Sexuality and $\mathrm{H}$ ealth Collective in Brazil (en anglais, portugais et espagnol), № 6, 1995.

Doing More with Less: The Marie Stopes Clinics of Sierra Leone (en anglais seulement), no 7, 1995.

Introducing Sexuality within Family Planning: Three Positive Experiences from Latin America and the Caribbean (en anglais et espagnol), no 8, 1997.
U sing CO PE to Improve Q uality of Care: The Experience of the Family Planning Association of Kenya (en anglais et espagnol), no 9 , 1998.

Alone You Are Nobody, Together We Float: The M anuela Ramos Movement (en anglais et espagnol), no 10, 2000.

From Patna to Paris: Providing Safe and $\mathrm{Hu}$ mane Abortion (en anglais seulement), $\mathrm{n} 011$, 2001.

U niversal Sexuality Education in Mongolia: Educating Today to Protect Tomorrow (en anglais seulement), no 12, 2002.

What about Us? Bringing Infertility into Reproductive Health Care (en anglais seulement), no 13, 2002.

"M y Father Didn't Think This Way": Nigerian Boys Contemplate Gender Equality (en anglais), no 14, 2003. 


\section{Q/C/Q n 14 français}

ualité/Quality /Calidad/Quali 